\title{
Determination of the Thickness and Composition Profiles for a Film of Binary Mixture on a Solid Substrate
}

\author{
$\check{\text { L. Fraštia }}^{1}$ *, U. Thiele ${ }^{1}$ and L. M. Pismen ${ }^{2}$ \\ ${ }^{1}$ Department of Mathematical Sciences, Loughborough University \\ Loughborough, Leicestershire, LE11 3TU, UK \\ ${ }^{2}$ Minerva Center for Nonlinear Physics of Complex Systems \\ Technion-Israel Institute of Technology, 32000 Haifa, Israel
}

\begin{abstract}
We determine the steady-state structures that result from liquid-liquid demixing in a free surface film of binary liquid on a solid substrate. The considered model corresponds to the static limit of the diffuse interface theory describing the phase separation process for a binary liquid (model-H), when supplemented by boundary conditions at the free surface and taking the influence of the solid substrate into account. The resulting variational problem is numerically solved employing a Finite Element Method on an adaptive grid. The developed numerical scheme allows us to obtain the coupled steady-state film thickness profile and the concentration profile inside the film. As an example we determine steady state profiles for a reflection-symmetric twodimensional droplet for various surface tensions of the film and various preferential attraction strength of one component to the substrate. We discuss the relation of the results of the present diffuse interface theory to the sharp interface limit and determine the effective interface tension of the diffuse interface by several means.
\end{abstract}

Key words: Cahn-Hilliard theory, model-H, phase separation, diffuse interface, phase-field model, demixing coupled to dewetting, surface evolution, variational method, FEM

AMS subject classification: 76B45, 74A50, 65N30

\footnotetext{
${ }^{*}$ Corresponding author. E-mail: L.Frastia@lboro.ac.uk
} 


\section{Introduction}

Simultaneous demixing and dewetting frequently occurs in industrial applications that involve films of soft matter on solid substrates. Examples include several coating technologies or the production of hierarchically structured functional polymer layers and printable polymeric electronic circuits. In emerging nano-technological applications, the thickness of the diffuse interface between different phases might become comparable to the structure size or layer thickness. This implies that the interaction between internal diffuse interfaces and sharp free surfaces and/or solid boundaries becomes an issue that has to be understood, in particular, for films of sub-micrometer thicknesses.

Various experimental studies as reviewed, e.g., in Ref. [13] involve films of phase separating polymers. The resulting structured films are often seen as resulting from either a dewetting process of the film or a phase separation of the components within the film. However, neither of the two descriptions is complete as the finally observed films show a modulated surface profile and a concentration profile within the film. Examples of final structures include drops of one component embedded in a film of the other component (that shows a surface modulation), and drops of one component on a film of the other component [13]. The aim of the present paper is to present a variational technique that allows us to describe such final steady state structures that might be reached through a number of dynamical pathways. For instance, dewetting induced by phase separation is studied in Ref. [26]; surface roughening of a phase-separated polymer mixture in Ref. [18]; wetting, phase separation, and the pathways to resulting structures of polymer mixtures are described in Refs. [44, 20,43]. The development of theoretical approaches to 'simple' phaseseparating films might in the future be extended to account for more involved situations like, for instance, evaporating films of solutions of binary mixtures on horizontal [16] or inclined [31] substrates.

Theoretical approaches to the demixing problem are commonly based on the Cahn-Hilliard theory [7, 27] using the Landau-Cahn free energy functional approximation to model phase separation in a bulk system while neglecting the effects of confinement. A small number of studies focuses on phase-separation in a gap between solid walls [9, 5, 25] or at a single rigid wall [8].

Such an approach is, however, not valid when at least one of the boundaries is a free surface, i.e., it corresponds to a film surface that is free to move and thereby to change its profile. To describe the time evolution of such a system the convective flow of the bulk liquid has to be accounted for. Then one may describe in a consistent and simultaneous manner the coupled effects of diffusive phase separation, surface and interface tensions, and hydrodynamic convective motion. The coupled transport equations for concentration (convective Cahn-Hilliard equation $[14,15]$ ) and momentum (Navier-Stokes-Korteweg equations) for a phase-separating binary liquid or liquidgas mixture are known as model-H [17]. Note that this and similar theories are often referred to as phase-field theories. Several groups use model-H to study the dynamics of fluids in the bulk or in a fixed confined geometry [1, 35, 19, 2, 41, 28, 42]. Recently, model-H was re-derived employing phenomenological non-equilibrium thermodynamics to consolidate a number of slightly differing formulations in the literature [40]. The model is supplemented by boundary conditions for velocity and concentration fields at the free surface and the solid substrate and is used to investigate steady 
stratified layers [40] and their linear stability with respect to lateral perturbations in the film thickness and concentration profiles [29]. A similar approach employing a simpler model was taken in Ref. [12].

For very thin films the time-scales for vertical and lateral structuring may separate. In consequence, one can assume that the film first phase-separates vertically on a short time-scale. Then the film evolves a horizontal structure on a longer time-scale. Under this circumstances an alternative approach is viable based on long-wave theory (or lubrication approximation) [33, 21]. In this approach the later stages of the evolution are studied as the evolution of a two-layer film on a solid substrate $[36,3,10,37,38]$, i.e., the diffuse interface of the vertical stratification is replaced by a sharp interface which is described by its height above the substrate $h_{i}$ in the same way as the local height of the film is $h$. Then the coupled evolution equations for the profiles $h_{i}(x, t)$ and $h(x, t)$ of the liquid-liquid and liquid-gas interfaces are determined. Extensions include the study of two-layer films with surfactants [11].

In the present work, we take a different approach. We do not restrict our attention to long-wave structures, but focus on the steady state equilibrium structures (film thickness and concentration profiles) that will result from the long-time evolution, i.e., after all short-time layering and longtime coarsening processes have let the system settle in an energy minimum. Such states are described by the static limit of model-H including the boundary conditions at the free surface and the substrate. The static limit can be obtained as solution of a variational problem (see appendix of [40]) - a formulation we employ here to numerically obtain the steady state solutions.

We discuss that variational formulation in Sections 2. and 3.. Thereby, we restrict our attention to a two-dimensional (2D) film on a solid substrate. To solve this model, in Section 4.1. we introduce a numerical procedure based on Finite Elements (FE). Steady state solutions for selected parameter values are computed and described in Section 4.2.. Thereby, a continuation procedure is employed where one uses either the surface tension of the free surface or the magnitude of the van der Waals interaction with the substrate as the continuation parameter. The results are discussed in Section 5.. In particular, we compare the numerical solutions with the limiting case of large surface tension using a one-dimensional theory for solutions with radial symmetry. We also discuss arc fitting procedures to obtain sharp interface equivalents for the diffuse interface solutions. Finally, the influence of the substrate is elucidated. The final Section 6. concludes and gives an outlook.

\section{The model for coupled demixing and dewetting}

The free energy functional for a partially miscible two-component liquid film on a solid substrate can be written in the form (cf. appendix of Ref. [40])

$$
\begin{aligned}
F\left[c, \Omega, \lambda_{\mathrm{d}}, \lambda\right] & =\int_{\Omega}\left[\frac{\sigma_{\mathrm{c}}}{2}(\nabla c)^{2}+f(c ; b, \boldsymbol{x})\right] \mathrm{d} V \\
& -\lambda_{\mathrm{d}}\left[\int_{\Omega} c_{1} \mathrm{~d} V-C_{1} V_{0}\right]-\lambda\left[\int_{\Omega} \mathrm{d} V-V_{0}\right]+\oint_{\partial \Omega} \gamma \mathrm{d} A .
\end{aligned}
$$

Here, $c_{i}=\rho_{i} /\left(\rho_{1}+\rho_{2}\right)$ and $C_{i}$ for $i=1,2$ are the dimensionless local and mean concentration of component $i$, respectively; $c=c_{1}-c_{2}=2 c_{1}-1$ is the difference of concentrations; $\sigma_{\mathrm{c}}$ is the 
effective rigidity related to the interface tension of the diffuse interface separating the phases (see next Section). $V_{0}$ is the prescribed volume of the domain $\Omega$ occupied by the liquid; $\gamma$ is the surface tension of the free surface that is here assumed not to depend on concentration (such a dependence will be considered elsewhere); $f(c ; b, \boldsymbol{x})$ is the volume density of the local Helmholtz free energy of the fluid. $\lambda$ and $\lambda_{\mathrm{d}}$ are the Lagrange multipliers enforcing the total volume to be $V_{0}$ and the total volume taken by the component 1 to be $C_{1} V_{0}$, respectively. The components 1 and 2 are locally pure when $c$ is 1 and -1 , respectively. The fluid is assumed to be incompressible and of constant density $\rho=\rho_{1}+\rho_{2}$. For the local free energy we assume the form

$$
f(c ; b, \boldsymbol{x})=-f_{0}\left[\varphi(c)-\frac{b c_{1}}{z^{3}}\right],
$$

with

$$
\varphi(c)=\ln \left(1-c^{2}\right)+q c^{2},
$$

where the $z$-axis is perpendicular to the planar substrate, and $q$ and $b$ are constants. The energy includes the van der Waals interaction with the substrate ( $z$-dependent term). The singular form of the function $\varphi(c)$, fashioned after the van der Waals equation of state, confines $c$ within the physical interval $-1<c<1$ and is useful for setting the boundary condition at the substrate, as specified below. Such a form for the energy $-f_{0} \phi(c)$ one obtains, e.g. using Flory-Huggins theory. For $q>1$, the symmetric function $-\varphi(c)$ has two minima corresponding to two phases. Here we consider $b>0$, i.e., component 1 is repelled by the substrate. The case $b<0$ is related by the symmetry $\left(b \rightarrow-b, c \rightarrow-c, C_{1} \rightarrow C_{2}\right)$.

Equation (2.1) is non-dimensionalised using $f_{0}$ as the energy scale and the characteristic thickness of the diffuse interface, $l_{c}=\sqrt{\sigma_{c_{1}} / f_{0}}=\sqrt{4 \sigma_{\mathrm{c}} / f_{0}}$, as the length scale. Further on, we shall numerically compute the film thickness profile $h(x)$ and the concentration profile $c(\boldsymbol{x})$ in a 2D layer on a solid substrate, that is infinitely extended and periodic with period $2 L$ along the $x$-direction. Retaining the same notation for the scaled (dimensionless) functions and variables, we rewrite Eq. (2.1) as

$$
\begin{aligned}
F\left[c(\boldsymbol{x}), h(x), \lambda_{\mathrm{d}}, \lambda\right] & =\int_{0}^{L} \int_{0}^{h(x)}\left[\frac{(\nabla c)^{2}}{8}+f(c ; b, \boldsymbol{x})-\lambda_{\mathrm{d}} c_{1}-\lambda\right] \mathrm{d} z \mathrm{~d} x \\
& +\gamma \int_{0}^{L} \sqrt{1+\left[h^{\prime}(x)\right]^{2}} \mathrm{~d} x+\lambda_{\mathrm{d}} C_{1} A_{0}+\lambda A_{0}
\end{aligned}
$$

where $\boldsymbol{x}=(x, z)$; and $A_{0}$ denotes the prescribed area in the considered 2D problem in analogy with $V_{0}$ in Eq. (2.1). Note, that the dimensionless surface tension $\gamma$ in (2.4) corresponds to the ratio of the dimensional surface tension and the internal interfacial tension, $\gamma_{\mathrm{i}}$, which is defined in Section 3.. To compute symmetric solutions, it is sufficient to consider half a period, i.e. $x \in[0, L]$, with the boundary conditions (BCs) $\partial_{x} c=h^{\prime}(x)=0$ at $x=0$ and $x=L$, that are already included as the natural BCs in our variational principle Eq. (2.4). For $b>0$, the substrate is shifted to $z=-d$ for numerical convenience, where $0<d \ll 1$, and the $\mathrm{BC}$ at $z=0$ is defined by the asymptotic condition $c_{1}(x, 0)=d^{3} / b$, which follows from Eq. (2.2). Note, however, that in calculations with $b>0, C_{1}, A_{0}$, and $h(x)$ correspond to the region of positive $z$ only. The $\mathrm{BC}$ for $c$ at the free surface 
is taken to be zero normal derivative, $\partial_{z} c-h^{\prime}(x) \partial_{x} c=0$, which corresponds to a surface tension that does not depend on concentration. Again, this is the natural BC contained already in Eq. (2.4). Similarly, for the case $b=0$ the natural $\mathrm{BC}$ at the substrate is $\partial_{z} c=0$. The more general case where one of the components is preferred at the free surface will be considered elsewhere.

\section{Basic equations}

First, we consider the basic phase-separating solution for a one-dimensional infinitely extended system. The spatial coordinate is $z$. Minimizing the functional (2.4), assuming $b=0$, results in the Euler-Lagrange equation

$$
c^{\prime \prime}(z)+4 \varphi^{\prime}(c(z))+2 \lambda_{\mathrm{d}}=0,
$$

subject to the natural boundary conditions $c^{\prime}(z)=0$ at $z \rightarrow \pm \infty$. Integrating Eq. (3.1), using the notation $c(z)=c_{ \pm}$for $z \rightarrow \pm \infty$, one obtains

$$
\frac{1}{2} c^{\prime 2}(z)=4\left[\varphi\left(c_{-}\right)-\varphi(c(z))\right]+2 \lambda_{\mathrm{d}}\left[c_{-}-c(z)\right] .
$$

Quadratures yield the profile $c(z)$ in the inverse form

$$
z=\int_{c(0)}^{c(z)} \frac{\mathrm{d} c}{c^{\prime}(c)}=\frac{1}{2} \int_{c(0)}^{c(z)} \frac{\mathrm{d} c}{\sqrt{2\left[\varphi\left(c_{-}\right)-\varphi(c)\right]+\lambda_{\mathrm{d}}\left(c_{-}-c\right)}} .
$$

The free energy function $-\varphi(c)$, defined in Eq. (2.3), is a symmetric double-well function admitting two stable phases with $c=c_{ \pm}$. For $\lambda_{\mathrm{d}}=0$, Eq. (3.1) has a simple kink solution with the average composition $C_{1}=1 / 2, c(0)=0$, and $c_{ \pm}= \pm \sqrt{1-1 / q}$. The interface tension of the diffuse interface is computed as

$$
\gamma_{\mathrm{i}}=\int_{-\infty}^{\infty} c_{1}^{\prime 2} \mathrm{~d} z=\frac{1}{4} \int_{-\infty}^{\infty} c^{\prime 2} \mathrm{~d} z=\frac{1}{4} \int_{c_{-}}^{c_{+}} c^{\prime}(c) \mathrm{d} c
$$

where one substitutes for $c^{\prime}$ from Eq. (3.2). In our numerical computations we used $q=2.5$, which gives $\gamma_{\mathrm{i}}=0.58909$ and $c_{ \pm}= \pm 0.77460$. The dimensional interfacial tension $\gamma_{i}^{\operatorname{dim}}$ is then $f_{0} l_{c} \gamma_{\mathrm{i}}$, where $f_{0}$ is the energy scale and $l_{c}=\sqrt{\sigma_{\mathrm{c}_{1}} / f_{0}}=\sqrt{4 \sigma_{\mathrm{c}} / f_{0}}$ is the width of the diffuse interface. Note that an estimation of the dimensional form of the integral (3.4) gives $\gamma_{i}^{\text {dim }} \sim \sigma_{\mathrm{c}} / l_{c}$. For a more extensive discussion of non-dimensionalisation in the context of model-H see [40, 29].

It is useful to indicate the diffuse interface between the two phases by a simple curve - the apparent sharp interface (ASI). As a natural generalization of the 1D case (cf. [34]) the Gibbs interface is defined for the general 3D case as the level set of $c(\boldsymbol{x})$ that separates domains at assumed constant $c=c_{ \pm}$in such a way that the same average concentration $V_{0}^{-1} \int_{\Omega} c_{1} \mathrm{~d} V=C_{1}$ is obtained as for the original diffuse interface. The Gibbs interface (a contour curve in our 2D case) is a one-parameter curve uniquely determined by the constant value of $c$ along it: $c=c_{\mathrm{G}}$. So, the number $c_{\mathrm{G}}$ is the solution of

$$
c_{+} A_{1}\left(c_{\mathrm{G}}\right)+c_{-} A_{2}\left(c_{\mathrm{G}}\right)=\int_{\Omega} c(\boldsymbol{x}) \mathrm{d} \boldsymbol{x}=C_{1} A_{0}
$$


where $A_{1}$ and $A_{2}$ denote the areas of the respective phases at $c_{+}$and $c_{-}$separated by the $c_{\mathrm{G}}$-contour. $A_{0}=A_{1}+A_{2}$ is the total area of the considered domain. For the kink solution $c(z)$ described here, Gibbs interface reduces to the point $z=0$, where $c(0)=c_{\mathrm{G}}=0$. In Section 5. other concepts are introduced to define curves as representatives of apparent sharp interfaces.

\section{Numerical 2D solution}

\subsection{Finite element variational method}

The $2 \mathrm{D}$ problem is solved by the finite element method (FEM). We introduce isoparametric finite elements (FE), i.e., we define (using matrix notation)

$$
\begin{aligned}
x & =\boldsymbol{H}(\boldsymbol{\xi}) \hat{\boldsymbol{x}}_{x}, & z & =\boldsymbol{H}(\boldsymbol{\xi}) \hat{\boldsymbol{x}}_{z}, \\
h & =\boldsymbol{H}\left(\boldsymbol{\xi}_{s}\left(\xi_{s}\right)\right) \hat{\boldsymbol{h}}=\boldsymbol{H}_{s}\left(\xi_{s}\right) \hat{\boldsymbol{h}}, & c & =\boldsymbol{H}(\xi, \eta) \hat{\boldsymbol{c}},
\end{aligned}
$$

where $\boldsymbol{H}$ is the $1 \times n$ (row) matrix of $n$ given basis functions corresponding to the chosen element type. $\boldsymbol{\xi}=(\xi, \eta)$ are the natural coordinates of the element, that usually belong to the range $[-1,1]$. In our calculations we used linear triangular elements with area coordinates, well known in the FEM literature [4]. The column vectors of the unknown nodal values are denoted by $\hat{\boldsymbol{x}}_{x}, \hat{\boldsymbol{x}}_{z}, \hat{\boldsymbol{h}}, \hat{\boldsymbol{c}}$. The variable $\boldsymbol{\xi}_{s}$ denotes the $\boldsymbol{\xi}$-curve that corresponds to the element edge that belongs to the top boundary $\partial \Omega^{\mathrm{t}}$. We are using isoparametric elements, i.e., $\boldsymbol{H}$ is identical for all fields.

Using the FE discretization, Eqs. (4.1) and (4.2), the variables $c$ and $h$ are expressed as functions of the FE natural coordinates $\xi, \eta$. The $x, z$ coordinates are themselves functions of $\xi, \eta$. The functional $F\left[c, h, \lambda_{\mathrm{d}}, \lambda\right]$ in Eq. (2.4), becomes

$$
\begin{aligned}
F & =F\left[c(\boldsymbol{\xi}), h\left(\xi_{s}\right), \lambda_{\mathrm{d}}, \lambda\right] \\
& =\int_{\Omega(\boldsymbol{\xi})}\left\{\frac{[\nabla c(\boldsymbol{\xi})]^{2}}{8}+f[c(\boldsymbol{\xi}) ; b, \boldsymbol{x}(\boldsymbol{\xi})]-\lambda_{\mathrm{d}} \frac{[c(\boldsymbol{\xi})+1]}{2}-\lambda\right\} J_{A}(\boldsymbol{\xi}) \mathrm{d}^{2} \boldsymbol{\xi} \\
& +\gamma \int_{\partial \Omega^{\mathrm{t}}\left(\xi_{s}\right)} J_{s}\left(\xi_{s}\right) \mathrm{d} \xi_{s}+\lambda_{\mathrm{d}} C_{1} A_{0}+\lambda A_{0},
\end{aligned}
$$

where $\nabla$ is the gradient in $\boldsymbol{x}$-space,

$$
J_{A}=\frac{\partial(x, z)}{\partial(\xi, \eta)}, \quad \text { and } \quad J_{s}=\frac{\mathrm{d} s}{\mathrm{~d} \xi_{s}} .
$$

In order to determine the stationarity condition for the functional (4.3), one needs to calculate $\delta F$. Using Eqs. (4.1) and (4.2) we note that

$$
\begin{aligned}
\delta x(\xi) & =\boldsymbol{H}(\boldsymbol{\xi}) \delta \hat{\boldsymbol{x}}_{x}, & & \delta z(\boldsymbol{\xi})=\boldsymbol{H}(\boldsymbol{\xi}) \delta \hat{\boldsymbol{x}}_{z}, \\
\delta h\left(\xi_{s}\right) & =\boldsymbol{H}_{s}\left(\xi_{s}\right) \delta \hat{\boldsymbol{h}}_{1} & & \delta c(\boldsymbol{\xi})=\boldsymbol{H}(\boldsymbol{\xi}) \delta \hat{\boldsymbol{c}} .
\end{aligned}
$$


Our FE mesh will change to accommodate different free surface profiles, i.e., $\boldsymbol{x}$ is adapted to $h(x)$. In order to define a unique FE discretization for arbitrarily varying $h(x)$, all degrees of freedom (DOFs) connected with the FE mesh, $\hat{\boldsymbol{x}}_{x}, \hat{\boldsymbol{x}}_{z}$, have to be expressed in terms of the DOFs of the variational problem, i.e., we have to define rules for mesh adaption.

For the present problem, it is advantageous to fix the $x$-coordinates of all nodes, $\hat{\boldsymbol{x}}_{x}$, and adapt only their $z$-coordinates, $\hat{\boldsymbol{x}}_{z}$, to the $h\left(\xi_{s}\right)$-profile. This is done in such a way that their height above the substrate measured in the local height of the profile $h(x)$ is constant, i.e., for the $i$ th node

$$
\hat{x}_{x i}=\mathrm{const}, \quad \hat{x}_{z i}=\frac{\hat{x}_{z 0 i}}{\boldsymbol{H}_{s}\left(\xi_{s}\left(\hat{x}_{x i}\right)\right) \hat{\boldsymbol{h}}_{0}} \boldsymbol{H}_{s}\left(\xi_{s}\left(\hat{x}_{x i}\right)\right) \hat{\boldsymbol{h}}, .
$$

where symbols $\hat{\boldsymbol{x}}_{z 0}, \hat{\boldsymbol{h}}_{0}$ denote variables $\hat{\boldsymbol{x}}_{z}, \hat{\boldsymbol{h}}$ in their given initial configuration. Eq. (4.6) defines the relation between $z$-coordinates of all nodes and the DOFs of the free surface. One can write it symbolically as

$$
\hat{\boldsymbol{x}}_{z}=\boldsymbol{Q} \hat{\boldsymbol{h}} .
$$

Using Eqs. (4.6), (4.7), and (4.1), we have

$$
\delta x=0, \quad \delta z=\boldsymbol{H}(\boldsymbol{\xi}) \delta \hat{\boldsymbol{x}}_{z}=\boldsymbol{H}(\boldsymbol{\xi}) \boldsymbol{Q} \delta \hat{\boldsymbol{h}} .
$$

Thus the independent DOFs of the functional $F$ are $\hat{\boldsymbol{c}}, \hat{\boldsymbol{h}}, \lambda_{\mathrm{d}}$, and $\lambda$. The gradient in Eq. (4.3) can be written as

$$
(\nabla c)(\boldsymbol{\xi})=\boldsymbol{T}^{\mathrm{T}}(\boldsymbol{\xi})\left[\partial_{\boldsymbol{\xi}} \boldsymbol{H}^{\mathrm{T}}(\boldsymbol{\xi})\right]^{\mathrm{T}} \hat{\boldsymbol{c}},
$$

where $\boldsymbol{T}(\boldsymbol{\xi})=\left(\partial_{\boldsymbol{\xi}} \boldsymbol{x}\right)^{-1}$ is the inverted transformation matrix $\partial_{\boldsymbol{\xi}} \boldsymbol{x}$ defined as

$$
\mathrm{d} \boldsymbol{x}=\left(\partial_{\boldsymbol{\xi}} \boldsymbol{x}\right) \mathrm{d} \boldsymbol{\xi}
$$

In the following derivation, we use index notation with Einstein's convention of summation. Eq. (4.9) reads

$$
\left(\nabla_{i} c\right)(\boldsymbol{\xi})=T_{j i}(\boldsymbol{\xi})\left[\partial_{\xi_{j}} H_{l}(\boldsymbol{\xi})\right] \hat{c}_{l} .
$$

The resulting variation of $\nabla c$ is

$$
\begin{aligned}
\delta\left(\nabla_{i} c\right) & =T_{j i}(\boldsymbol{\xi})\left[\partial_{\xi_{j}} H_{l}(\boldsymbol{\xi})\right] \delta \hat{c}_{l}-T_{j 2}(\boldsymbol{\xi})\left(\partial_{\xi_{n}} H_{m}\right) Q_{m l} \delta \hat{h}_{l} T_{n i}(\boldsymbol{\xi})\left[\partial_{\xi_{j}} H_{k}(\boldsymbol{\xi})\right] \hat{c}_{k} \\
& =\left(\nabla_{i} H_{l}\right) \delta \hat{c}_{l}-\left(\nabla_{2} H_{k}\right) \hat{c}_{k}\left(\nabla_{i} H_{m}\right) Q_{m l} \delta \hat{h}_{l},
\end{aligned}
$$

where $\nabla_{2} \equiv \partial_{z}$. In matrix notation we have

$$
\delta(\nabla c)=(\nabla \boldsymbol{H}) \delta \hat{\boldsymbol{c}}-\left(\partial_{z} \boldsymbol{H}\right) \hat{\boldsymbol{c}}(\nabla \boldsymbol{H}) \boldsymbol{Q} \delta \hat{\boldsymbol{h}}=(\nabla \boldsymbol{H}) \delta \hat{\boldsymbol{c}}-\left(\partial_{z} c\right)(\nabla \boldsymbol{H}) \boldsymbol{Q} \delta \hat{\boldsymbol{h}}
$$

The variations of the Jacobians are

$$
\begin{aligned}
\delta J_{A} & =\partial_{\xi} x \partial_{\eta} \delta z-\partial_{\eta} x \partial_{\xi} \delta z=\left(\partial_{\xi} x \partial_{\eta} H_{i}-\partial_{\eta} x \partial_{\xi} H_{i}\right) Q_{i j} \delta \hat{h}_{j} \\
& =J_{A}\left(\partial_{z} H_{i}\right) Q_{i j} \delta \hat{h}_{j}=J_{A}\left(\partial_{z} \boldsymbol{H}\right) \boldsymbol{Q} \delta \hat{\boldsymbol{h}}, \\
\delta J_{s} & =J_{s}^{-1} \partial_{\xi_{s}} h\left(\xi_{s}\right) \partial_{\xi_{s}} \delta h\left(\xi_{s}\right)=J_{s}^{-1} \partial_{\xi_{s}} \boldsymbol{H}_{s} \hat{\boldsymbol{h}} \partial_{\xi_{s}} \boldsymbol{H}_{s} \delta \hat{\boldsymbol{h}} .
\end{aligned}
$$


The first row of Eq. (4.14) shows that $\delta J_{A}$ is independent of the DOFs of the variational problem for our rules of mesh adaption. Finally, the stationarity condition for the functional (4.3) is

$$
\begin{aligned}
\delta F & =\delta F\left[\hat{\boldsymbol{c}}, \hat{\boldsymbol{h}}, \lambda_{\mathrm{d}}, \lambda\right]=\delta \hat{\boldsymbol{c}}^{\mathrm{T}} \int_{\Omega}\left[\frac{1}{4}(\nabla \boldsymbol{H})^{\mathrm{T}} \nabla c+\boldsymbol{H}^{\mathrm{T}}\left(\partial_{c} f(c ; b, \boldsymbol{x})-\lambda_{\mathrm{d}} / 2\right)\right] \mathrm{d}^{2} \boldsymbol{\xi} \\
& +\delta \hat{\boldsymbol{h}}^{\mathrm{T}} \boldsymbol{Q}^{\mathrm{T}} \int_{\Omega}\left[-\frac{1}{4}(\nabla \boldsymbol{H})^{\mathrm{T}}(\nabla c) \partial_{z} c+\boldsymbol{H}^{\mathrm{T}} \partial_{z} f(c ; b, \boldsymbol{x})\right] J_{A} \mathrm{~d}^{2} \boldsymbol{\xi} \\
& -\delta \lambda_{\mathrm{d}} \int_{\Omega} c_{1} \mathrm{~d}^{2} \boldsymbol{\xi}-\delta \lambda \int_{0}^{L} h(x) \mathrm{d} x \\
& +\delta \hat{\boldsymbol{h}}^{\mathrm{T}} \boldsymbol{Q}^{\mathrm{T}} \int_{\Omega}\left(\partial_{z} \boldsymbol{H}\right)^{\mathrm{T}} J_{A}\left[\frac{(\nabla c)^{2}}{8}+f(c ; b, \boldsymbol{x})-\lambda_{\mathrm{d}} c_{1}-\lambda\right] \mathrm{d}^{2} \boldsymbol{\xi} \\
& +\delta \hat{\boldsymbol{h}}^{\mathrm{T}} \gamma \int_{\partial \Omega^{\mathrm{t}}}\left(\partial_{\xi_{s}} \boldsymbol{H}_{s}\right)^{\mathrm{T}}\left(\partial_{\xi_{s}} \boldsymbol{H}_{s}\right) \hat{\boldsymbol{h}} J_{s}^{-1} \mathrm{~d} \xi_{s}+\delta \lambda_{\mathrm{d}} C_{1} A_{0}+\delta \lambda A_{0}=0,
\end{aligned}
$$

which gives the nonlinear FE equations of the problem. Newton's method is used to solve the resulting system of equations numerically.

\subsection{Computational procedure and parameters}

We numerically investigate small steady two-dimensional droplets, i.e., solutions with a relatively small and compact spatial domain of phase 1 within the ambient liquid composed of phase 2 or, at the interface of phase 2 and air. The geometric parameters are fixed to $L=13.75$ (lateral system size) and $\bar{h}=5.5$ (mean film thickness).

The continuation procedure is as follows

1. The basic mesh is created with a maximum element edge size of 0.55 , i.e., one has about 10 elements along the vertical of the original rectangular domain and about 25 elements along the horizontal. As detailed below, a drop attached to the free surface is used as a starting solution or initial approximation on the coarse mesh.

2. The coarse mesh is used to effectively obtain the indicative numerical solution for a given value of the continuation parameter $(\gamma$ or $b)$.

3. The obtained solution on the coarse mesh is used as an initial approximation for the adaptive mesh refinement and further computation of the refined solution. The refinement is then repeated once more and the final solution is obtained. The refinement predominantly takes place in the diffuse interface region, in the region close to the substrate, and in zones along the free surface, where high concentration gradients and/or surface curvature are detected. In regions where both refinement steps are performed, the typical size of a final element is 0.1375 , i.e., one has about 7 elements per characteristic width of the diffuse interface. A typical refined mesh and sample solutions are shown in Figs. 1 and 2, respectively. 
4. The continuation parameter is advanced and the previous solution on the basic coarse mesh, obtained in the "coarse" step 2, is used as an initial approximation and steps 2 to 4 are repeated.

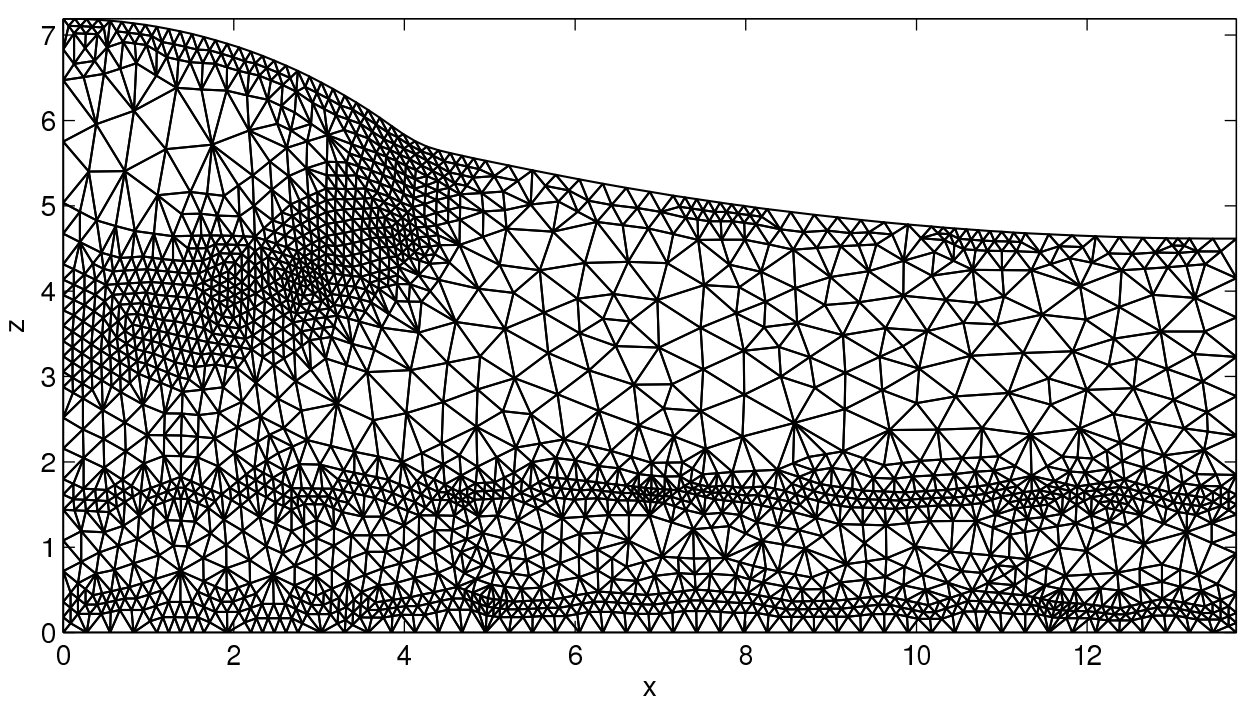

Figure 1: Steady state droplet geometry with the refined mesh for the solution at $\gamma=1.3, b=10$. Refined regions are encountered at the diffuse interface, at the boundary of the depletion zone (described in Section 5.5. and shown below in Fig 10), and near the highly curved parts of the free surface.

In the limit of large surface tension $\gamma \gg 1$, i.e. when the ratio between the free surface tension and interfacial tension becomes very large, in the sharp interface approximation, the energy is minimal for a droplet with a semi-circular interphase boundary attached either to the substrate or to the almost planar free surface. The latter has to be chosen as a starting point for the determination of droplets that are repelled by the substrate. This is here always the case.

Normally, we fix $b$ and gradually decrease $\gamma$. Thereby, we generate a series of solutions with increasing curvature $\kappa_{\mathrm{S}}$ of the free surface and decreasing curvature of the interphase boundary ( $\kappa_{\mathrm{G}}$, as represented by the Gibbs interface). The case $b=0$ is investigated to compute positions of the Gibbs interface and the average curvatures (which may be distorted by the influence of the substrate). The breakdown of the formula we use to determine Gibbs interface (3.5) is illustrated by a series of solutions obtained by continuation in $b$ at fixed $\gamma$. Also here, the solutions on the coarse basic mesh, for $b=0$ are used as an initial approximation. In all calculations, the remaining parameters are fixed at $q=2.5, A_{0}=L \bar{h}=75.627$, and $C_{1}=0.2$. In addition, for $b>0$ we use $d=0.055$. In the following section we analyse the obtained symmetric $2 \mathrm{D}$ solutions.

In physical terms the discussed drops correspond to two-dimensional nano-droplets. For diffuse interface widths between $10 \mathrm{~nm}$ and $100 \mathrm{~nm}$ the chosen mean film thickness [lateral domain size] lies roughly between $50 \mathrm{~nm}$ and $0.5 \mu \mathrm{m}$ [150nm and $1.5 \mu \mathrm{m}]-$ typical ranges in Ref. [13]. The studied range of the dimensionless surface tension $\gamma$ (ratio of dimensional surface tension to dimensional effective interface tension of the diffuse interface) is between $O(1)$ and $O\left(10^{2}\right)$. The 


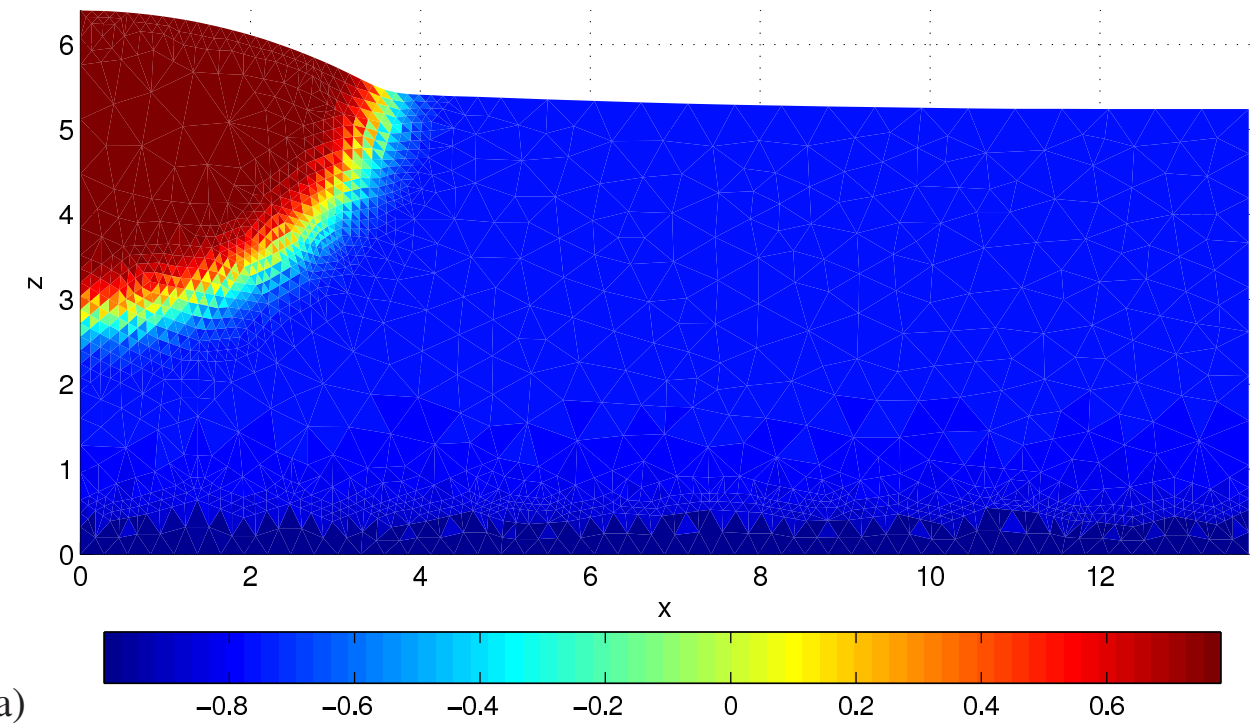

(a)

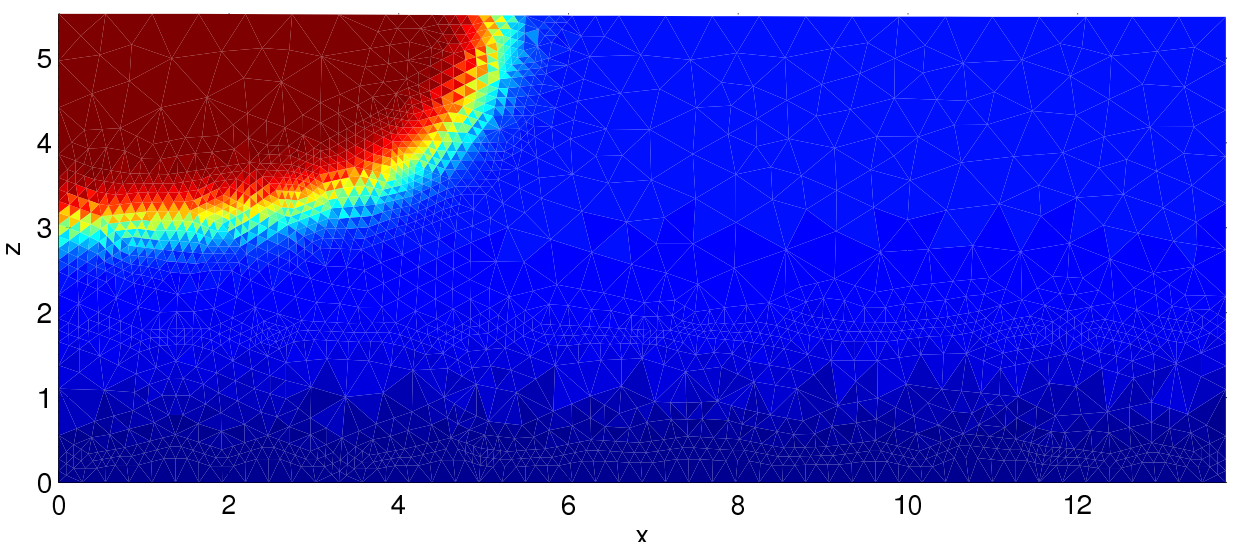

(b)

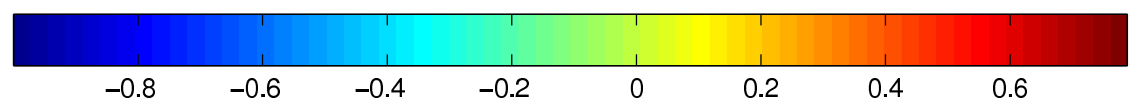

Figure 2: Concentration $c(\boldsymbol{x})$ and thickness $h(x)$ profiles for (a) $\gamma=1.2, b=1$ and (b) $\gamma=178$, $b=14$. The significant repulsion of component 1 by the substrate and the small $\gamma$ displace a lensshaped drop in (a). At very large $b$ and $\gamma$ one observes a very squeezed drop and a large depletion layer in (b). 


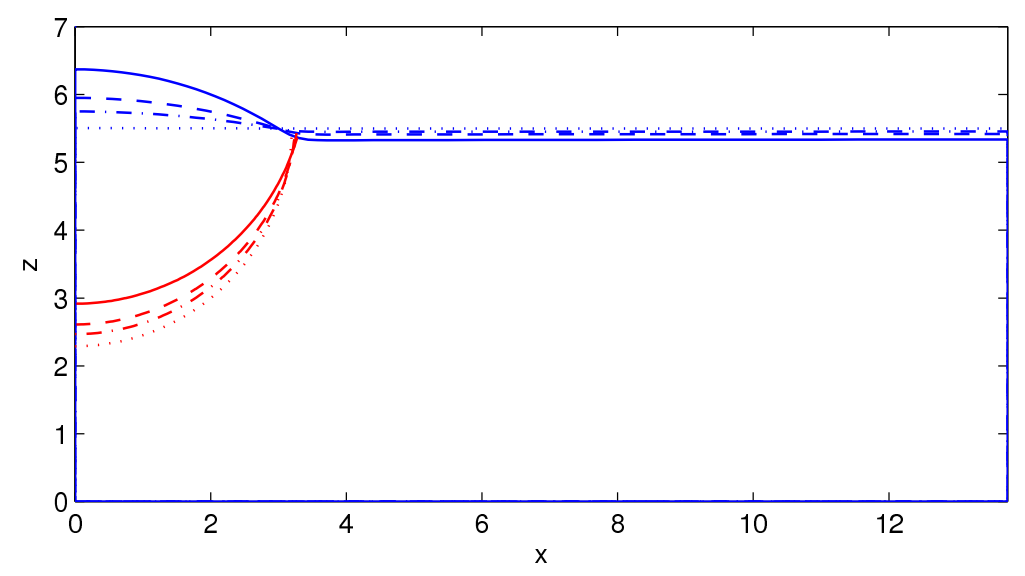

Figure 3: Superimposed droplet shapes for increasing $\gamma$ at fixed $b=0$. In particular, shown are profiles for $\gamma=0.95$ (solid line), $\gamma=1.78$ (dashed line), $\gamma=3.16$ (dot-dashed line), and $\gamma=178$ (dotted line). The diffuse interface is represented by the Gibbs interface as described in Section 5.1..

latter corresponds to the limit of large surface tension $\gamma \gg 1$, e.g., to two polymers that are 'nearly' miscible: they demix with a very wide diffuse interface. The lower considered limit of $\gamma \approx 1$ corresponds to a rather strong interfacial tension: the components can be considered to be strongly immiscible; the diffuse interface is then very thin (about $1 \mathrm{~nm}$ ). For two polymers one would normally expect $\gamma \approx 10$, i.e., their interfacial tension is about one order of magnitude smaller than their polymer-air surface tension. A further discussion of typical parameter values can be found in [29].

\section{Results and discussion}

\subsection{Properties of the diffuse interface}

The sequence of droplet shapes for neutral substrates $(b=0)$ shown in Fig. 3 is obtained by gradually lowering $\gamma$. In the figure, the diffuse interface is represented by Gibbs interface at $c_{\mathrm{G}}$ [defined by Eq. (3.5)].

The value of $c_{\mathrm{G}}$ may be influenced by the substrate, as discussed in Section 5.5.. In addition, for large $b, c_{\mathrm{G}}$ is very close to $c_{+}$and the $c_{\mathrm{G}}$-contour no longer represents the drop region. Moreover, it may become intractable numerically. There are other ways to define an apparent sharp interface (ASI) but none of them is equivalent to the diffuse interface. A very convenient way is to define the ASI as the contour of maximal excess of the local free energy $\max [f(c ; 0, \boldsymbol{x})] \equiv \max [-\varphi(c)]$ which is at $c=c_{\mathrm{e}}=0$.

Yet another ASI can be constructed using force-balance arguments. The stress in the considered 
binary fluid, [1, 40, 35], is given by

$$
\begin{aligned}
\boldsymbol{\tau} & =\left[f\left(2 c_{1}-1 ; b, \boldsymbol{x}\right)+\frac{\left(\nabla c_{1}\right)^{2}}{2}-\lambda_{\mathrm{d}} c_{1}-\lambda\right] \boldsymbol{I}-\left(\nabla c_{1}\right)\left(\nabla c_{1}\right) \\
& =\left[f(c ; b, \boldsymbol{x})+\frac{(\nabla c)^{2}}{8}-\lambda_{\mathrm{d}} \frac{(c+1)}{2}-\lambda\right] \boldsymbol{I}-\frac{1}{4}(\nabla c)(\nabla c) .
\end{aligned}
$$

For a static solution one has

$$
\nabla \cdot \boldsymbol{\tau}=0
$$

For $b=0$, taking the sharp interface limit the stress distribution reduces to constant pressures inside and outside of the drop and constant interface force located at the sharp interphase boundary (cf. [19]). Eq. (5.2) indicates that the difference between the pressure inside and outside of the drop - the Laplace pressure - is related to the constant interface tension $\gamma_{i}$ [defined by Eq. (3.4)], and the local curvature of the sharp interface $\kappa_{\mathrm{i}}$ by

$$
p_{\text {in }}-p_{\text {out }}=\gamma_{\mathrm{i}} \kappa_{\mathrm{i}} \text {. }
$$

Eq. (5.3) implies $\kappa_{\mathrm{i}}=$ const, i.e., the possible static solutions are circular drops of one phase completely immersed in the other phase, lens-shaped drops attached to the free surface, and semicircular drops attached to an unbiased substrate.

Let us consider a ridge (2D drop) with the lens-shaped cross section. The surface and interfacial tensions at the contact point, where the ASI meets with the free surface, have to be in equilibrium

$$
\gamma \boldsymbol{t}_{1}+\gamma \boldsymbol{t}_{2}+\gamma_{\mathrm{P}} \boldsymbol{t}_{\mathrm{P}}=\mathbf{0}
$$

Here, $\gamma$ and $\gamma_{\mathrm{P}}$ are the surface and interface tension for the free surface and the ASI, respectively. The $t_{1}, t_{2}$, and $t_{\mathrm{P}}$ are corresponding tangent vectors at the contact point. Eq. (5.4) represents Plateau's law. Note, that projecting Eq. (5.4) onto the $z$-axis and neglecting the component $t_{2 z}$, we obtain for the present geometry

$$
\gamma \kappa_{\mathrm{s}}=\gamma_{\mathrm{P}} \kappa_{\mathrm{P}}
$$

which is the condition of equal Laplace pressures that determines curvatures for the specified surface tensions. For $b>0$, the component $t_{2 z}$ cannot be neglected and one has to use the full $z$-component of Eq. (5.4). This is done in all calculations below in Section 5.3., where the forcebalance approach and Eq. (5.4) are employed to determine the ASI. Using force-balance arguments, one can also uniquely replace the original diffuse interface by the ASI in a way that is presented in the following section, where the semi-circular drop is examined.

\subsection{Semi-circular droplets in the limit of large surface tension}

Consider a 2D droplet located at the free surface. For $\gamma \gg 1$ the surface is nearly flat and one has $h(x) \approx \bar{h}$. For a distant and noninteracting substrate, this droplet can be approximated by a semi-circular droplet. It can be analyzed using polar coordinates with the origin at the center of the circle, which lies on the axis of lateral reflection symmetry at $x=0$. 


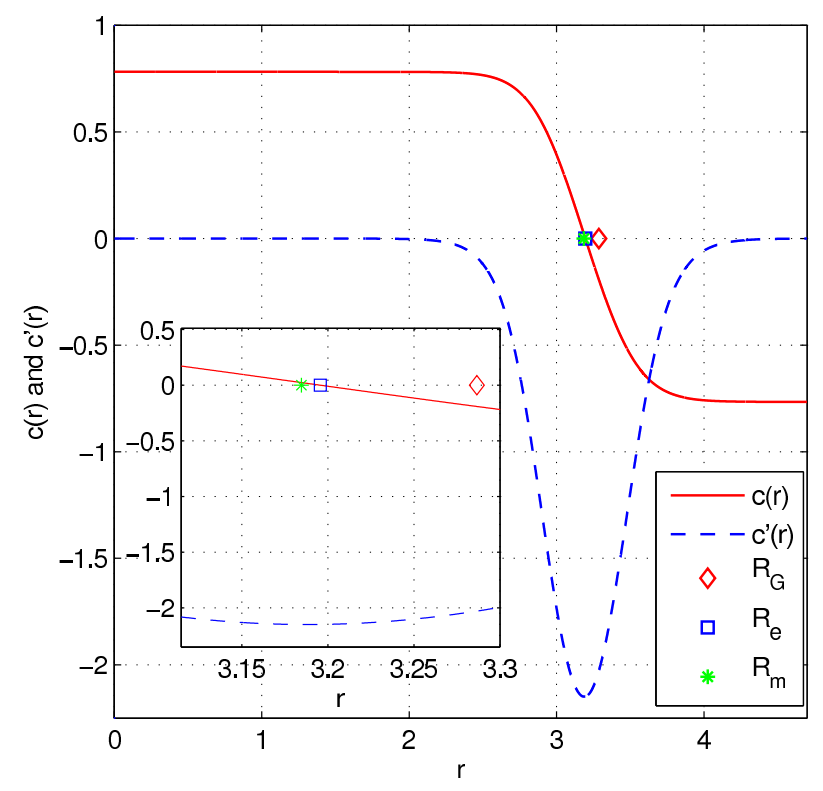

Figure 4: Shown are the characteristics of solutions with (semi-)circular symmetry. The main graph gives the concentration field $c=c(r)$ and its derivative $c^{\prime}(r)$. The inset presents with a higher resolution the positions of the radii $R_{\mathrm{G}}, R_{\mathrm{e}}$, and $R_{\mathrm{m}}$ corresponding to the Gibbs interface, the interface of maximal excess free energy, and the interface determined by force-balance arguments, respectively. Details of their definition as well as a detailed discussion can be found in Section 5.2..

The Euler-Lagrange equation arising from the variation of the functional (2.4) with respect to $\delta c$, rewritten in polar coordinates $(r, \theta)$ and assuming circular symmetry, reads

$$
c^{\prime \prime}(r)+4 \frac{c^{\prime}(r)}{r}+4 \varphi^{\prime}(c(r))+2 \lambda_{\mathrm{d}}=0
$$

The asymptotic condition at $r \rightarrow \infty$ is $c^{\prime}(r)=0$. In this case, one cannot obtain the first integral in a closed form. The profile $c(r)$ is obtained by numerically solving the nonlinear ODE (5.6) as an initial value problem with initial conditions

$$
c(r)=c_{0}, c^{\prime}(r)=0 \quad \text { at } \quad r=0 .
$$

Using a shooting method one finds a $c_{0}$ such that one obtains a solution with one diffuse interface and with $c^{\prime}(r)$ approaching 0 for $r$ larger than the radius of a drop. Results are shown in Fig. 4. The parameter values used are the same as for the 2D FEM solution (see Section 4.2.). There, however, one additionally uses $b=0, \gamma=178$ and obtains the corresponding $\lambda_{\mathrm{d}}=0.23841$ (here we use $\lambda_{\mathrm{d}}=0.238$ which gives $\left.C_{1}=0.1996\right)$. Then the present $1 \mathrm{D}$ solution is close to the $2 \mathrm{D}$ FEM solution and both can be compared.

The values of $c_{\mathrm{G}}$ and $R_{\mathrm{G}}=\kappa_{\mathrm{G}}{ }^{-1}$ can be calculated using the volume balance formula (3.5) 
written for circular symmetry

$$
\left(c_{+}-c_{-}\right) \pi R_{\mathrm{G}}^{2}=\int_{0}^{L}\left[c(r)-c_{-}\right] 2 \pi r \mathrm{~d} r, \quad \text { with } \quad L=\sqrt{\frac{4 A_{0}}{\pi}},
$$

where $A_{0}=L \bar{h}$ is the prescribed volume in $2 \mathrm{D}$ calculations, see Section 4.2 .. The factor 4 in Eq. (5.8) is motivated by the fact that the considered 2D solution resembles one quarter of a circular drop. We obtain $\kappa_{\mathrm{G}}=0.30427$ and $c_{\mathrm{G}}=-0.19152$ (cf. Fig. 4). Maximal excess of the free energy at $c_{\mathrm{e}}=0$ gives $\kappa_{\mathrm{e}}=0.31293$.

Using force-balance arguments, we determine the ASI to be located at the radius $r=R_{\mathrm{m}}$, where the 0th and the 1 st moment of $\tau_{\theta \theta}$, [given by Eqs. (5.1) and (5.2)] are equivalent to the corresponding moments of the traction for the fictitious drop with sharp interface at the ASI, i.e.,

$$
\begin{gathered}
\int_{0}^{L} \tau_{\theta \theta} \mathrm{d} r=-p_{\mathrm{L}} R_{\mathrm{m}}+\gamma_{\mathrm{m}}=0 \\
\int_{0}^{L} \tau_{\theta \theta} r \mathrm{~d} r=-\frac{p_{\mathrm{L}} R_{\mathrm{m}}^{2}}{2}+\gamma_{\mathrm{m}} R_{\mathrm{m}}
\end{gathered}
$$

These two equations are written assuming zero pressure in the ambient phase 2, which can be checked by computing the 1.h.s. of Eq. (5.9). The solution of these equations is

$$
\begin{aligned}
\gamma_{\mathrm{m}} & =p_{\mathrm{L}} R_{\mathrm{m}} \\
\text { and } \quad R_{\mathrm{m}} & =\sqrt{\frac{2}{p_{\mathrm{L}}} \int_{0}^{L} \tau_{\theta \theta} r \mathrm{~d} r}
\end{aligned}
$$

where the Laplace pressure can be calculated as the difference of the pressures at the center and far outside the drop, where $\nabla c$ is negligible. Using Eq. (5.1) we obtain

$$
p_{\mathrm{L}}=f\left(c_{\text {out }}\right)-f\left(c_{\text {in }}\right)+\left(c_{\text {in }}-c_{\text {out }}\right) \lambda_{\mathrm{d}} / 2 \text {. }
$$

Using the numerical values obtained for the 1D solution $c_{\text {in }}=c_{0}$ and $c_{\text {out }}=c(L)$, we obtain $p_{\mathrm{L}}=0.18432$. Finally, we compute from Eq. (5.12) $\kappa_{\mathrm{m}}=0.31401$ and $c\left(R_{\mathrm{m}}\right)=0.023689$ (see Fig. 4). The interface tension is estimated as $\gamma_{\mathrm{m}}=p_{\mathrm{L}} / \kappa_{\mathrm{m}}=0.58700$. This value is somehow smaller than the nominal value $\gamma_{i}$, which was obtained from Eq. (3.4).

\subsection{Least squares curves fit}

Next, we compute the average curvatures directly from the obtained FE solutions. Then the interfacial tension of the diffuse interface can be approximated by $\gamma_{\mathrm{P}}$ using the force balance at the apparent contact point according to Eq. (5.4). This formula is analogous to the Young-Laplace law of balance of surface tensions, that enables one to calculate equilibrium contact angles of two fluid media or phases of a fluid at a substrate. In order to determine the equilibrium contact angles in a similar way, we perform a least squares fit of the drop boundary by two circular arcs and fit 
(b)

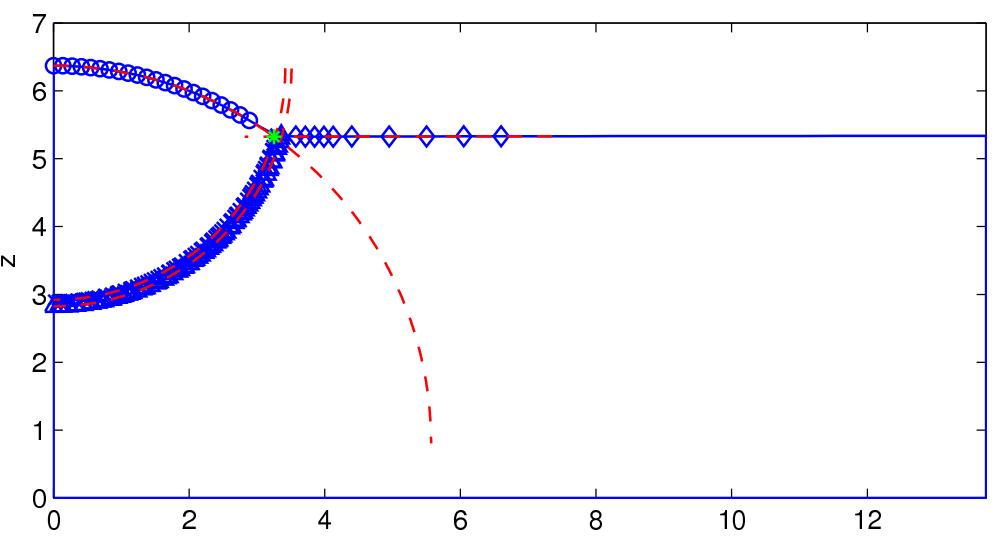

(a)

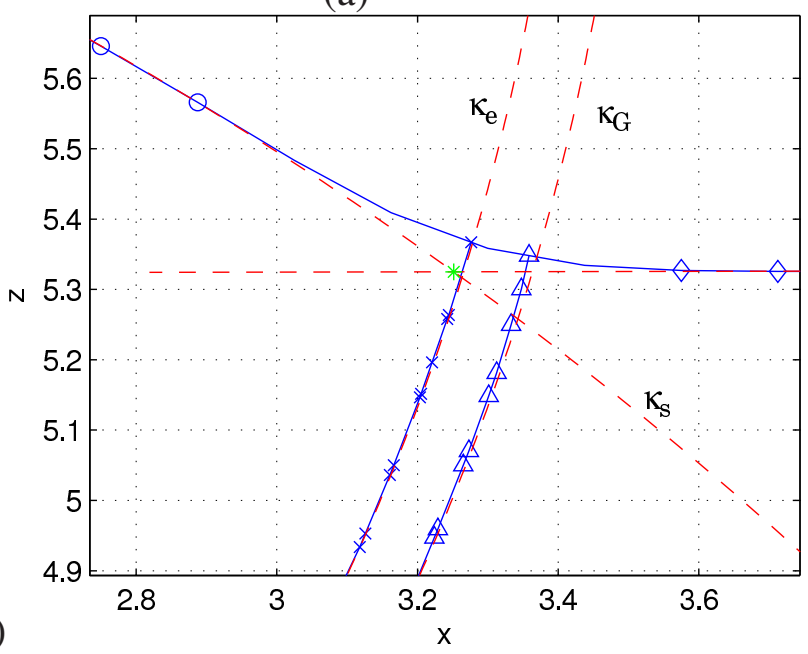

(c)

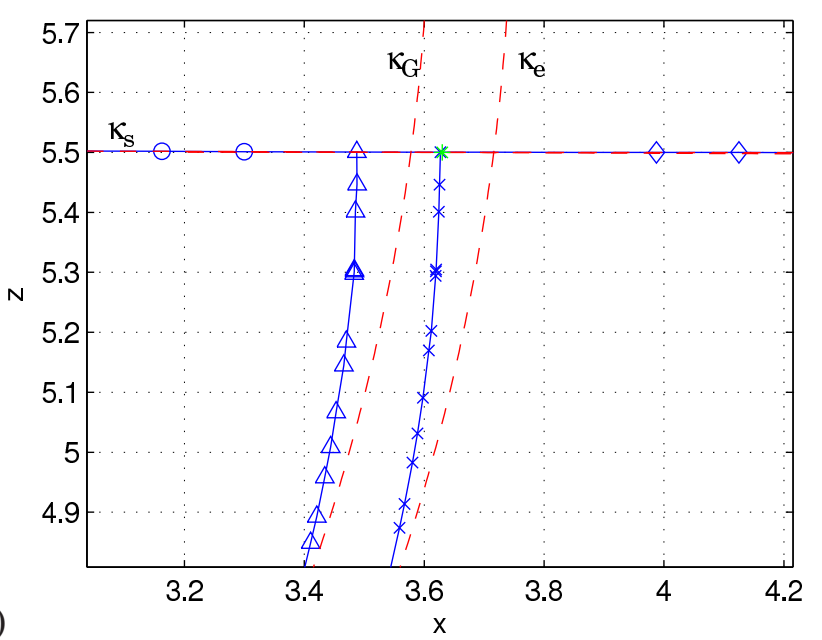

Figure 5: Least squares curves fit of the droplet solution. Circles and diamonds denote the datapoints for the droplet and film part of the free surface, respectively. Crosses and triangles denote the interfaces defined via the maximal excess free energy and the Gibbs criterion, respectively. Panel (a) gives the complete solution for $\gamma=0.95$ and $b=0$ whereas in (b) a corresponding zoom is given for the contact zone. Panel (c) shows a similar detail for $\gamma=178$ and $b=1$. Note, that the fitted arcs are denoted by their respective curvatures (cf. Section 5.3.). 
(a)
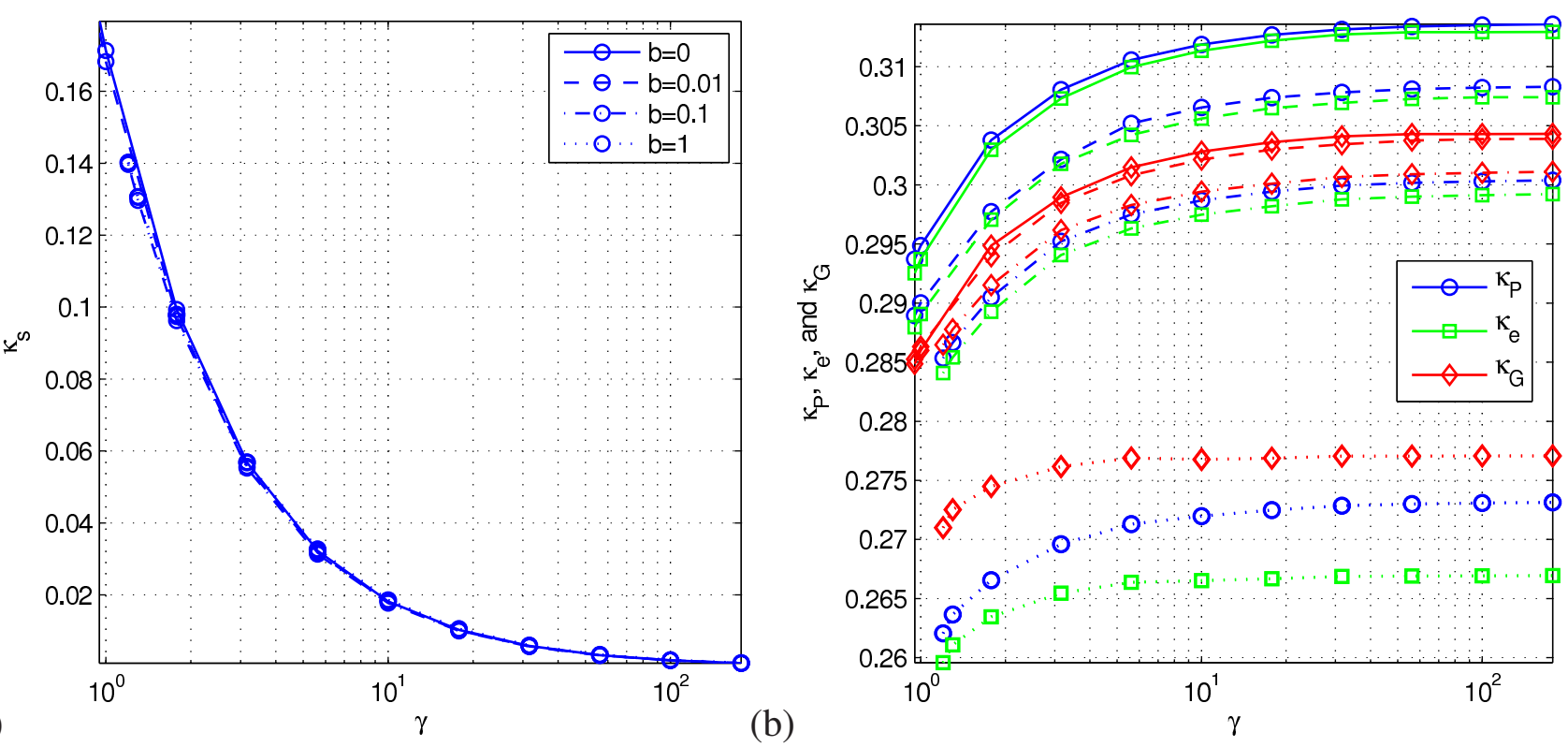

Figure 6: Dependences of the average curvatures (determined by least squares fitting) on $\gamma$ for $b=0,0.01,0.1$, and 1.0. The same line styles are used for both figures (a) and (b). (a) Average curvature $\kappa_{\mathrm{S}}$ of the free surface of the drop. (b) Average curvatures of various curves characterizing the diffuse interface (see Section 5.3. for details). $\kappa_{\mathrm{e}}$ and $\kappa_{\mathrm{G}}$ correspond to maximal excess free energy interface and Gibbs interface, respectively. $\kappa_{\mathrm{P}}$ corresponds to the arc passing through the contact point.

the free surface of phase 2 by a straight line. The data-points in the diffuse interface region are not used for fitting the free surface. Fig. 5 illustrates the curve fitting procedure. The intersection of the arc fitting the drop surface and the straight line determines both the apparent contact point and the vectors $t_{1}$ and $t_{2}$. It can be seen, that the arc fitted to the Gibbs interface (data-points denoted by triangles) does not pass through the contact point. This indicates that the Gibbs interface is a poor choice if one wants to effectively replace the actual nonlocal interface tension by the concentrated force - interface tension - acting on some sharp interface. A better choice is the contour of maximal excess of the local free energy as defined in Section 5.1. (crosses in Fig. 5). For our calculations, we determine the unit vector $\boldsymbol{t}_{\mathrm{P}}$ as the tangent to the arc concentric to the arc of maximum excess energy passing through the apparent contact point (this arc is not shown in the figure). The curvature of the latter arc is $\kappa_{\mathrm{P}}$, that of the maximal excess free energy arc is $\kappa_{\mathrm{e}}$, and that of the Gibbs arc is $\kappa_{\mathrm{G}}$. The dependencies of these curvatures on $\gamma$ for some fixed values of $b$ are shown in Fig. 6.

For the solution at $\gamma=178$ and $b=0$, i.e., corresponding to the 1D solution considered in Section 5.2., the curvatures are $\kappa_{\mathrm{G}}=0.30431, \kappa_{\mathrm{e}}=0.31293$, and $\kappa_{\mathrm{P}}=0.31360$ (cf. Fig. 6) and $c_{\mathrm{G}}=-0.18922$ (cf. Fig. 9b. Comparison with the results obtained in the 1D limiting case (Section 5.2.) shows good agreement.

Assuming that $\gamma$ is given and all angles are determined via fitted arcs, we use the $z$-component 
of Eq. (5.4) to calculate $\gamma_{\mathrm{P}}$ and the $x$-component to determine the error

$$
E=\left|\frac{\gamma t_{1 x}+\gamma t_{2 x}+\gamma_{\mathrm{P}} t_{\mathrm{P} x}}{\gamma_{\mathrm{P}}}\right|,
$$

for any value of $b$. For $b=0$, averaging over the results for $\gamma_{\mathrm{P}}$ ranging from 0.95 to 178 , we obtain the mean value $\gamma_{\mathrm{P}}=0.58426$. The largest error is $E=0.00617$. Note, that the interface tension $\gamma_{\mathrm{P}}$ increases with $b$, e.g. at $b=1$ we obtain the mean value $\gamma_{\mathrm{P}}=0.60505$ with maximal $E=0.02742$.

Note, that the interface tension $\gamma_{\mathrm{P}}$ for the $2 \mathrm{D}$ solutions is slightly smaller than the nominal value, $\gamma_{i}$, given by Eq. (3.4). This is due to the finite area of the FE region considered and the used numerical discretization.

\subsection{Influence of the substrate}

For $b>0$, the shape of the droplet is influenced by the substrate. Component 1 and therefore the droplet is repelled by the substrate and the shape of all interfaces may significantly deviate from arcs and straight lines.

The basic geometrical parameters of the droplet solutions (as characterized by the Gibbs contour) are the maximal thickness of the liquid film $h_{\max }$, the minimal height of the Gibbs contour $z_{\mathrm{Gmin}}$, and the vertical and horizontal extensions of the symmetric droplet $a_{\mathrm{d}}$ and $b_{\mathrm{d}}$, respectively. The ratio $a_{\mathrm{d}} / b_{\mathrm{d}}$ is the dimensionless form factor characterizing the shape of a droplet.

For the three non-zero fixed values $b=0.01,0.1$, and 1 , the surface tension $\gamma$ is gradually decreased to determine the dependencies of the droplet shape parameters on $\gamma$ shown in Figs. 7 and 8 . The asymptotic boundary condition at the substrate was used as described earlier in Section 2.. The volume and the average concentration were fixed at the same values as before. Curve fitting is illustrated in Fig. 5c, clearly indicating deviations between data-points and fitted arcs.

Inspecting Figs. 7 and 8 two effects can be discerned. First, with increasing $b$ and $\gamma$ the droplet is squeezed from below by the substrate and from above by the surface tension. Second, the droplet region with prevailing component 1 is as a whole repelled more strongly than the film with prevailing component 2, i.e., the droplet is lifted up as a whole, especially at higher $b$ and smaller $\gamma$. Figure 7 shows mainly the effect of the repelling of the whole drop, whereas Fig. 8 better reflects the changing shape of the droplet itself. The most rounded (squeezed) shape one finds at smallest (largest) $\gamma$ and $b$.

For the one-dimensional $c(z)$ profile determined in Section 3. the equilibrium chemical potential is $\lambda_{\mathrm{d}}=0$. Here, in order to stabilize a drop one needs to impose a nonzero $\lambda_{\mathrm{d}}$ that balances the Laplace pressure. This balance is expressed by Eq. (5.13), where the main contribution on the r.h.s. comes from the $\lambda_{\mathrm{d}}$-term. The dependence of the equilibrium chemical potential $\lambda_{\mathrm{d}}$ on $\gamma$ is shown for various $b$ in Fig. 9a. It can be seen that $\lambda_{\mathrm{d}}$ increases with increasing $\gamma$ and $b$, i.e., it is larger for a more squeezed drop. 
(a)

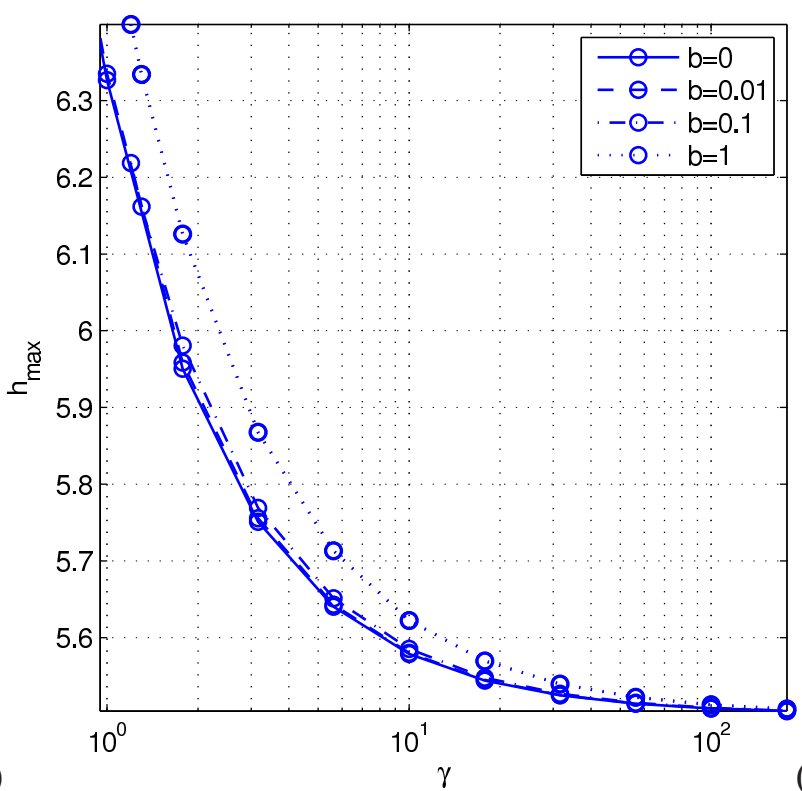

(b)

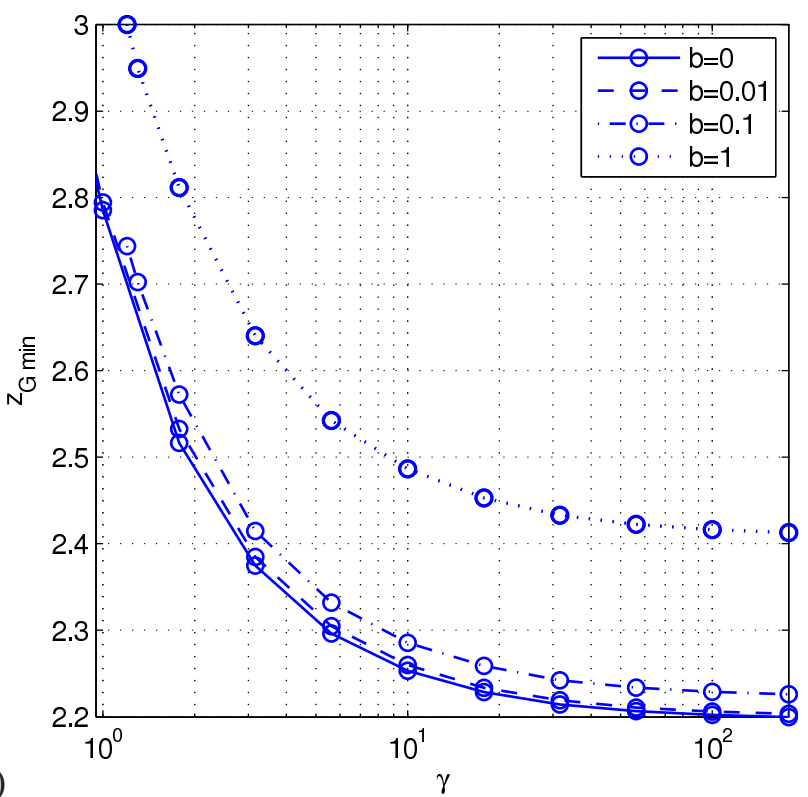

Figure 7: Shown are characteristic vertical measures as functions of $\gamma$ for $b=0,0.01,0.1$, and 1.0. (a) Height over $x=0$ of the maximum of the droplet ( $\left.h_{\max }\right)$. (b) Height over $x=0$ of the minimum of the Gibbs interface $\left(z_{\mathrm{G} \text { min }}\right)$.
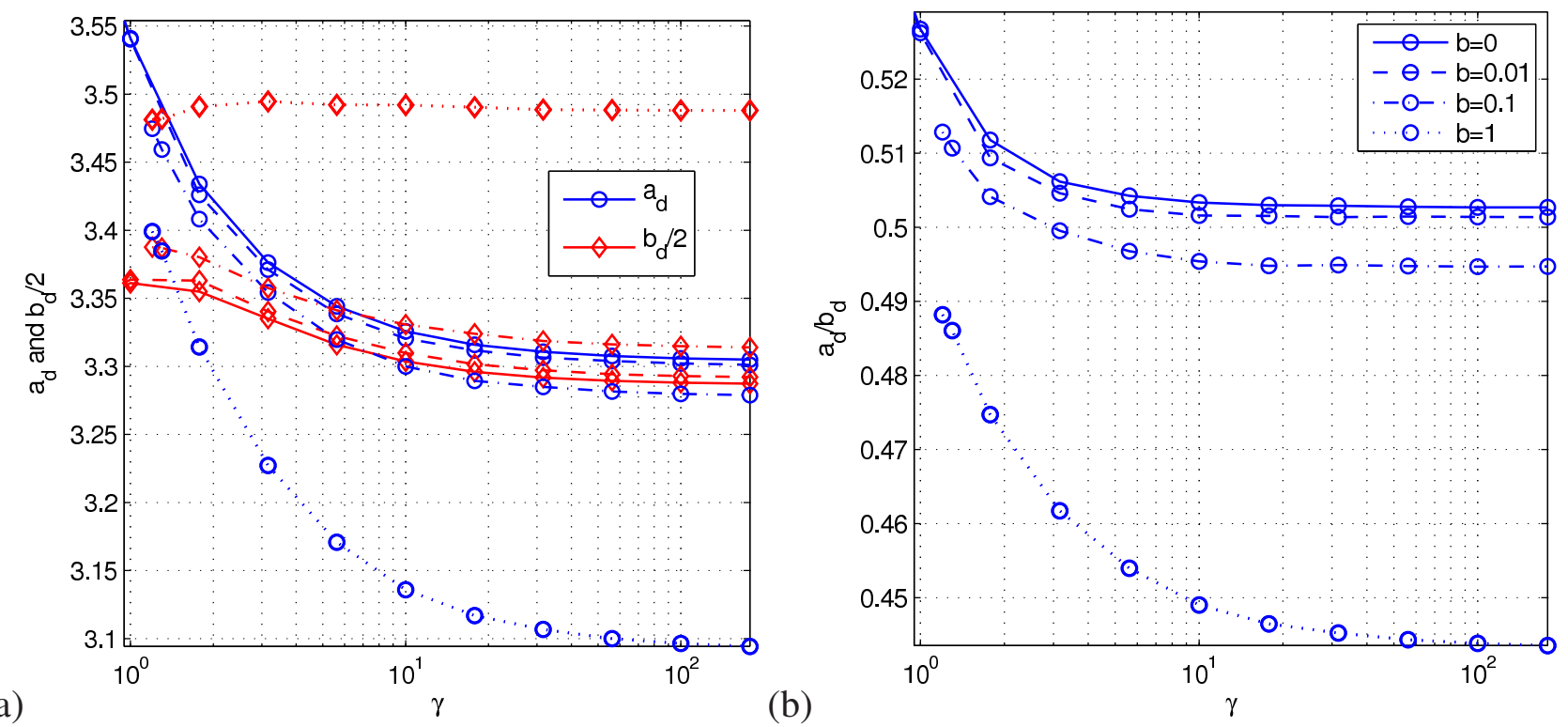

Figure 8: Shown are (a) the vertical and horizontal extensions of the drops as well as (b) their form factors as functions of $\gamma$ for $b=0,0.01,0.1$, and 1.0. The same line styles are used for both panels (a) and (b). Panel (a) gives the vertical size $a_{\mathrm{d}}=h_{\max }-z_{\mathrm{G} \text { min }}$ and the horizontal half-width of the droplet $b_{\mathrm{d}} / 2$ (as obtained from the Gibbs interface). Panel (b) gives the form factor $a_{\mathrm{d}} / b_{\mathrm{d}}$. 
(a)

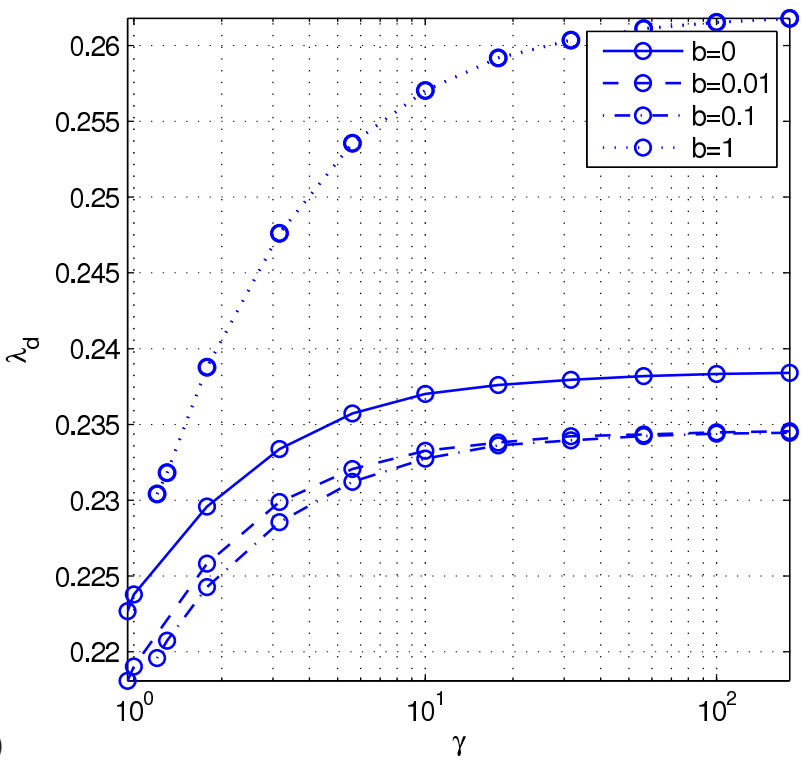

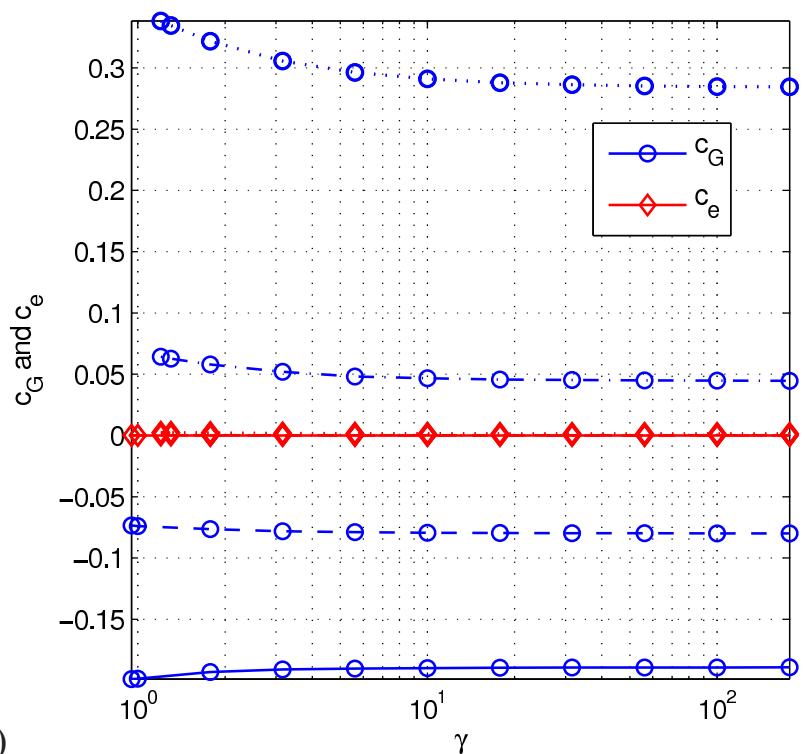

Figure 9: (a) Equilibrium chemical potential $\lambda_{\mathrm{d}}$. (b) Concentrations $c_{\mathrm{G}}$ (at the contour representing the Gibbs interface) and $c_{\mathrm{e}}=0$ (at the contour of maximal excess free energy). Shown are $\lambda_{\mathrm{d}}, c_{\mathrm{G}}$, and $c_{\mathrm{e}}$ as functions of $\gamma$ for $b=0,0.01,0.1$, and 1.0. The line styles on (a) and (b) are the same.

(a)

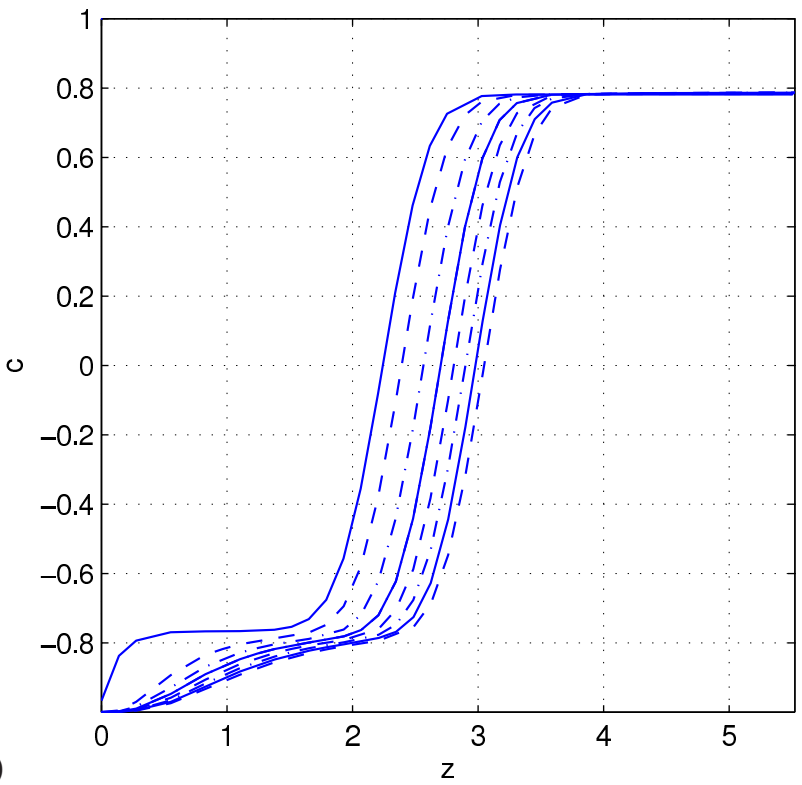

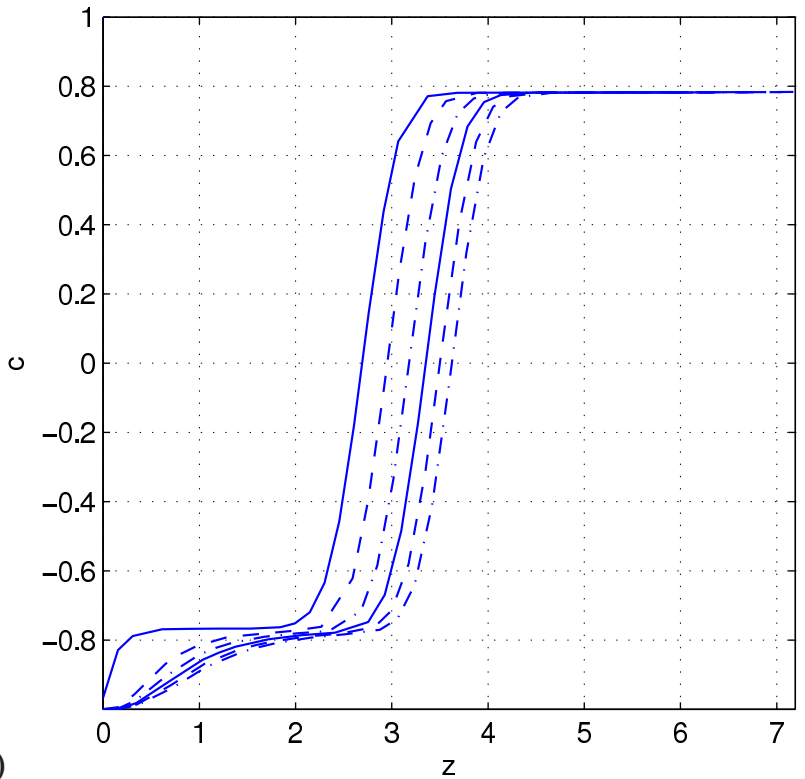

Figure 10: Shown are vertical concentration profiles $c(0, z)$ along the symmetry axis of the drop. Profiles for (a) $\gamma=178$ and $b=0.01,2,4,6,8,10,12$, and 14, with line styles: solid, dashed, dot-dashed, solid, dashed, ..., respectively; and (b) $\gamma=1.3$ and $b=0.01,2,4,6,8$, and 10 (line styles as in (a)). The formation of the depletion layer with diminished $c_{1}$ is particularly strong in (a) for $b=14$. 
(a)
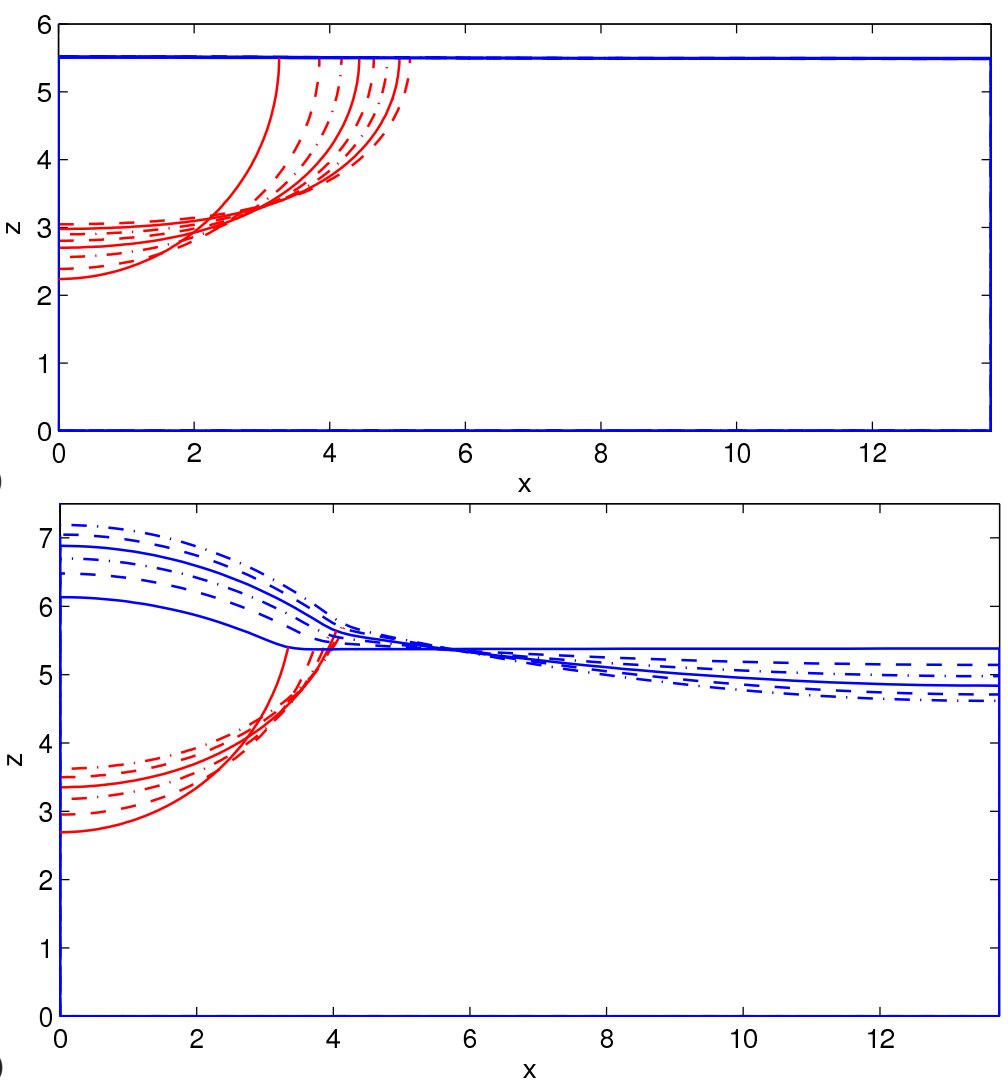

Figure 11: Shown are superimposed droplet and surface shapes for (a) $\gamma=178$ and $b=0.01,2$, $4,6,8,10,12$, and 14 ; and (b) $\gamma=1.3$ and $b=0.01,2,4,6,8$, and 10 . The line styles correspond to Fig. 10. In contrast to Fig. 3, here the diffuse interface is represented by the contour of maximal excess free energy at $c=c_{\mathrm{e}}=0$.

\subsection{Depletion layer and breakdown of the volume criterion}

An additional effect of the interaction with the substrate can be appreciated in Figs. $9 \mathrm{~b}$ and 10, that give the Gibbs curvature $c_{\mathrm{G}}(\gamma)$ for several $b$ and several vertical concentration profiles $c(0, z)$ on the symmetry axis of the droplet, respectively. In particular, in Fig. 10 one observes close to the substrate the formation of a depletion layer where component 1 is strongly repelled and component 2 is attracted. For $b=2$ the layer roughly covers the region $0<z<0.5$. The layer can be thought of as a "new phase" that is not included in the volume balance formula (3.5). The latter then increasingly exaggerates $c_{\mathrm{G}}$ with increasing $b$. As can be seen in Fig. 9b, $c_{\mathrm{G}}$ may be significantly larger than the value at maximum excess energy, $c_{\mathrm{e}}=0$. For even larger $b, c_{\mathrm{G}}$ is so close to $c_{+}$that the $c_{\mathrm{G}}$-contour may be intractable computationally. In this regime the Gibbs contour no longer represents the interface. Some droplet shapes in this regime are shown in Fig. 11, where the $c_{\mathrm{e}}$-contour is used to represent the interphase boundary.

In Section 5.4. we argue that $\lambda_{\mathrm{d}}$ increases with increasing $\gamma$ and $b$. Actually, as one can see in 
the corresponding Fig. 9a, for very small $b=0$ and 0.01 the graphs of $\lambda_{\mathrm{d}}(\gamma)$ are slightly shifted upwards with respect to their expected location. This is due to the (nearly) absent depletion layer (cf. Fig. 10). If such a layer were present the rest of the film would be slightly enriched with the repelled component 1 . This in turn would help to stabilize the droplet and lower the imposed $\lambda_{\mathrm{d}}$. Thus the effect of the $b$-term in the free energy (2.2) is twofold: (1) it increases the concentration of component 1 in the film above the depletion layer and (2) it repels the entire droplet of phase 1 when $b$ is sufficiently large.

\section{Conclusions}

In this contribution we have numerically examined the steady-state structures that result from liquid-liquid demixing in a two-dimensional free surface film of binary liquid on a solid substrate. The considered model corresponds to the static limit of the diffuse interface theory describing the phase separation process for a binary liquid (model-H). It has a variational structure, i.e., it can be directly derived as a variation of the underlying free energy functional. The model has been solved numerically employing a Finite Element Method on an adaptive grid and allows to obtain the coupled steady-state film thickness profile and the concentration profile inside the two-dimensional film. As an example we have determined steady state profiles for a symmetric droplet for various surface tensions of the film and various preferential attraction strength $b$ of one component to the substrate. For a neutral substrate $(b=0)$, in particular, we have obtained film thickness and concentration profiles as a function of surface tension. We have evaluated the relation of our diffuse interface result to the corresponding sharp interface solutions and solutions in the limit of large surface tension. As a result, we have shown that the computed curvatures of one-dimensional radially symmetric solutions and the corresponding two-dimensional FEM solution are in rather good agreement. The contact point (a notion of the sharp interface model) determined by forcebalance arguments for the one-dimensional case corresponds to the contact point determined by curve fitting in the two-dimensional case.

The interface tension of the diffuse interface between drop and liquid layer has been calculated by several independent means: (i) a nominal formula for infinitely extended phases gives $\gamma_{i}$; (ii) using the correspondence of the solution with $\gamma \gg 1$ to a solution of circular symmetry gives $\gamma_{\mathrm{m}}$; and (iii) using Plateau's law gives $\gamma_{\mathrm{P}}$. The values thus obtained are in good correspondence. Furthermore, we have observed changes in the vertical droplet position and droplet morphology due to a repulsive van der Waals interaction between component 1 of the binary liquid and the substrate. The results elucidate the structure of two-dimensional droplets of nanometric size in films of sub-micron thicknesses when phase separation is strongly influenced by boundary effects. Future extensions could focus on nanodroplets with radial symmetry and the general three-dimensional case. This would then allow us to analyse line tension effects.

Finally, it was shown that the sharp interface representation by an apparent sharp interface (ASI) depends on the particular criterion used: Gibbs volume balance, Plateau's law for surface tensions, or the maximal excess free energy $c$-contour. In consequence, we have concluded that in physical situations where the interface width is of the same order of magnitude as the mean film 
height, the nonlocal diffuse interface can normally not be reduced to a single sharp interface. A future study should take into account the depletion layer at the substrate by modifying Eq. (3.5) or deriving an alternative criterion for the determination of the Gibbs interface.

In the present study, we have used a generic expression for the local energy that takes into account van der Waals interactions between the two components of the liquid as well as the repulsion of one of the components by the substrate. This should, however, be seen as an example as the present computational approach can be directly adapted to various different physical settings. For instance, one may (i) employ expressions for the bulk free energy density that are adapted to particular polymer blends, (ii) incorporate a concentration-dependent surface tension at the free surface as in [40,29] to model situations where solutal Marangoni forces are present, (iii) apply the approach to films of liquid crystals by replacing the used scalar order parameter (concentration) by a vectorial or tensorial one (director orientation), (iv) incorporate wetting interactions of the mixture with the substrate. That would allow to investigate coupled dewetting of the film and decomposition inside the film. Further studies may also (v) consider substrates with heterogeneities, i.e., with topographical or chemical variations. Although in the latter case experimental results exist for binary mixtures $[24,32,13]$, theoretical results are at present only available for films of simple liquids $[22,23,6,39,30]$.

\section{Acknowledgements}

We acknowledge support by the Israel Science Foundation under grant \# 55/02 and by the European Union under grant PITN-GA-2008-214919 (MULTIFLOW).

\section{References}

[1] D.M. Anderson, G.B. McFadden, A.A. Wheeler. Diffuse-Interface methods in fluid mechanics. Ann. Rev. Fluid Mech., 30 (1998), 139-165.

[2] L.K. Antanovskii. Microscale theory of surface tension. Phys. Rev. E, 54 (1996), 6285-6290.

[3] D. Bandyopadhyay, R. Gulabani, A. Sharma. Stability and dynamics of bilayers. Ind. Eng. Chem. Res., 44 (2005), 1259-1272.

[4] K.-J. Bathe. Finite element procedures. Prentice-Hall, New Jersey, 2nd edition, 1995.

[5] K. Binder. Spinodal decomposition in confined geometry. J. Non-Equilib. Thermodyn., 23 (1998), 1-44.

[6] L. Brusch, H. Kühne, U. Thiele, M. Bär. Dewetting of thin films on heterogeneous substrates: Pinning vs. coarsening. Phys. Rev. E, 66 (2002), 011602.

[7] J.W. Cahn, J.E. Hilliard. Free energy of a nonuniform System. 1. Interfacual free energy. J. Chem. Phys., 28 (1958), 258-267. 
[8] H.P. Fischer, P. Maass, W. Dieterich. Novel surface modes in spinodal decomposition. Phys. Rev. Lett., 79 (1997), 893-896.

[9] H.P. Fischer, P. Maass, W. Dieterich. Diverging time and length scales of spinodal decomposition modes in thin films. Europhys. Lett., 42 (1998), 49-54.

[10] L.S. Fisher, A.A. Golovin. Nonlinear stability analysis of a two-layer thin liquid film: Dewetting and autophobic behavior. J. Colloid Interface Sci., 291 (2005), 515-528.

[11] L.S. Fisher, A.A. Golovin. Instability of a two-layer thin liquid film with surfactants: Dewetting waves. J. Colloid Interface Sci., 307 (2007), 203-214.

[12] O.A. Frolovskaya, A.A. Nepomnyashchy, A. Oron, A.A. Golovin. Stability of a two-layer binary-fluid system with a diffuse interface. Phys. Fluids, 20 (2008), 112105.

[13] M. Geoghegan, G. Krausch. Wetting at polymer surfaces and interfaces. Prog. Polym. Sci., 28 (2003), 261-302.

[14] A.A. Golovin, S.H. Davis, A.A. Nepomnyashchy. A convective Cahn-Hilliard model for the formation of facets and corners in crystal growth. Physica D, 122 (1998), 202-230.

[15] A.A. Golovin, A.A. Nepomnyashchy, S.H. Davis, M.A. Zaks. Convective Cahn-Hilliard models: From coarsening to roughening. Phys. Rev. Lett., 86 (2001), 1550-1553.

[16] L.V. Govor, J. Parisi, G.H. Bauer, G. Reiter. Instability and droplet formation in evaporating thin films of a binary solution. Phys. Rev. E, 71 (2005), 051603.

[17] P.C. Hohenberg, B.I. Halperin. Theory of dynamic critical phenomena. Rev. Mod. Phys., 49 (1977), 435-479.

[18] K.D. Jandt, J. Heier, F.S. Bates, E.J. Kramer. Transient surface roughening of thin films of phase separating polymer mixtures. Langmuir, 12 (1996), 3716-3720.

[19] D. Jasnow, J. Viñals. Coarse-grained description of thermo-capillary flow. Phys. Fluids, 8 (1996), 660-669.

[20] R.A.L. Jones, L.J. Norton, E.J. Kramer, F.S. Bates, P. Wiltzius. Surface-directed spinodal decomposition. Phys. Rev. Lett., 66 (1991), 1326-1329.

[21] S. Kalliadasis, U. Thiele (eds.). Thin Films of Soft Matter. Springer, Wien / New York, CISM 490, 2007.

[22] K. Kargupta, R. Konnur, A. Sharma. Instability and pattern formation in thin liquid films on chemically heterogeneous substrates. Langmuir, 16 (2000), 10243-10253.

[23] K. Kargupta, A. Sharma. Templating of thin films induced by dewetting on patterned surfaces. Phys. Rev. Lett., 86 (2001), 4536-4539. 
[24] A. Karim, J.F. Douglas, B.P. Lee, S.C. Glotzer, J.A. Rogers, R.J. Jackman, E.J. Amis, G.M. Whitesides. Phase separation of ultrathin polymer-blend films on patterned substrates. Phys. Rev. E, 57 (1998), R6273-R6276.

[25] R. Kenzler, F. Eurich, P. Maass, B. Rinn, J. Schropp, E. Bohl, W. Dieterich. Phase separation in confined geometries: Solving the Cahn-Hilliard equation with generic boundary conditions. Comp. Phys. Comm., 133 (2001), 139-157.

[26] T. Kerle, J. Klein, R. Yerushalmi-Rozen. Accelerated rupture at the liquid/liquid interface. Langmuir, 18 (2002), 10146-10154.

[27] J.S. Langer. An introduction to the kinetics of first-order phase transitions. in 'Solids far from Equilibrium' (ed. by Godreche), Cambridge University Press, (1992), 297-363.

[28] J. Lowengrub, L. Truskinovsky. Quasi-incompressible Cahn-Hilliard fluids and topological transitions. Proc. R. Soc. London Ser. A-Math. Phys. Eng. Sci., 454 (1998), 2617-2654.

[29] S. Madruga, U. Thiele. Decomposition driven interface evolution for layers of binary mixtures: II. Influence of convective transport on linear stability. Phys. Fluids, 21 (2009), 062104.

[30] S. Mechkov, M. Rauscher, S. Dietrich. Stability of liquid ridges on chemical micro- and nanostripes. Phys. Rev. E, 77 (2008), 061605.

[31] P. Müller-Buschbaum, E. Bauer, S. Pfister, S.V. Roth, M. Burghammer, C. Riekel, C. David, $\mathrm{U}$. Thiele. Creation of multi-scale stripe-like patterns in thin polymer blend films. Europhys. Lett., 73 (2006), 35-41.

[32] G. Nisato, B.D. Ermi, J.F. Douglas, A. Karim. Excitation of surface deformation modes of a phase-separating polymer blend on a patterned substrate. Macromolecules, 32 (1999), 2356-2364.

[33] A. Oron, S.H. Davis, S.G. Bankoff. Long-scale evolution of thin liquid films. Rev. Mod. Phys., 69 (1997), 931-980.

[34] L.M. Pismen. Mesoscopic hydrodynamics of contact line motion. Colloid Surf. APhysicochem. Eng. Asp., 206 (2002), 11-30.

[35] L.M. Pismen, Y. Pomeau. Disjoining potential and spreading of thin liquid layers in the diffuse interface model coupled to hydrodynamics. Phys. Rev. E, 62 (2000), 2480-2492.

[36] A. Pototsky, M. Bestehorn, D. Merkt, U. Thiele. Alternative pathways of dewetting for a thin liquid two-layer film. Phys. Rev. E, 70 (2004), 025201(R).

[37] A. Pototsky, M. Bestehorn, D. Merkt, U. Thiele. Morphology changes in the evolution of liquid two-layer films. J. Chem. Phys., 122 (2005), 224711. 
[38] A. Pototsky, M. Bestehorn, D. Merkt, U. Thiele. 3D Surface Patterns in liquid two-layer films. Europhys. Lett., 74 (2006), 665-671.

[39] U. Thiele, L. Brusch, M. Bestehorn, M. Bär. Modelling thin-film dewetting on structured substrates and templates: Bifurcation analysis and numerical simulations. Eur. Phys. J. E, 11 (2003), 255-271.

[40] U. Thiele, S. Madruga, L. Frastia. Decomposition driven interface evolution for layers of binary mixtures: I. Model derivation and stratified base states. Phys. Fluids, 19 (2007), 122106.

[41] N. Vladimirova, A. Malagoli, R. Mauri. Diffusion-driven phase separation of deeply quenched mixtures. Phys. Rev. E, 58 (1998), 7691-7699.

[42] N. Vladimirova, A. Malagoli, R. Mauri. Two-dimensional model of phase segregation in liquid binary mixtures. Phys. Rev. E, 60 (1999), 6968-6977.

[43] H. Wang, R.J. Composto. Thin film polymer blends undergoing phase separation and wetting: Identification of early, intermediate, and late stages. J. Chem. Phys., 113 (2000), 1038610397.

[44] H. Wang, R.J. Composto. Understanding morphology evolution and roughening in phaseseparating thin-film polymer blends. Europhys. Lett., 50 (2000), 622-627. 